21 \# both contributed equally to this work

* Address Correspondence to:

\title{
IFITM proteins promote SARS-CoV-2 infection and are targets for virus inhibition
}

\author{
Caterina Prelli Bozzo ${ }^{1 \#}$, Rayhane Nchioua ${ }^{1 \#}$, Meta Volcic ${ }^{1}$, Jana Krüger ${ }^{2}$, Sandra Heller ${ }^{2}$, \\ Christina M. Stürzel ${ }^{1}$, Dorota Kmiec ${ }^{1,3}$, Carina Conzelmann ${ }^{1}$, Janis Müller ${ }^{1}$, Fabian Zech ${ }^{1}$, \\ Desiree Schütz ${ }^{1}$, Lennart Koepke ${ }^{1}$, Elisabeth Braun ${ }^{1}$, Rüdiger Groß ${ }^{1}$, Lukas Wettstein ${ }^{1}$, \\ Tatjana Weil ${ }^{1}$, Johanna Weiß ${ }^{1}$, Daniel Sauter ${ }^{1,4}$, Jan Münch ${ }^{1}$, Federica Diofano ${ }^{5}$, Christine \\ Goffinet $^{6}$, Alberto Catanese ${ }^{7}$, Michael Schön ${ }^{7}$, Tobias Böckers ${ }^{7}$, Steffen Stenger ${ }^{8}$, Kei Sato ${ }^{9}$, \\ Steffen Just ${ }^{5}$, Alexander Kleger ${ }^{2}$, Konstantin M.J. Sparrer ${ }^{1 *}$ and Frank Kirchhoff ${ }^{1^{*}}$
}

${ }^{1}$ Institute of Molecular Virology, Ulm University Medical Centre, 89081 Ulm, Germany.

${ }^{2}$ Department of Internal Medicine I, Ulm University Medical Centre, 89081 Ulm, Germany.

${ }^{3}$ Department of Infectious Diseases, King's College London, WC2R 2LS London, United Kingdom. ${ }^{4}$ Institute of Medical Virology and Epidemiology of Viral Diseases, University Hospital Tübingen, 72076 Tübingen, Germany. ${ }^{5}$ Department of Internal Medicine II (Cardiology), Ulm University, 89081 Ulm, Germany. ${ }^{6}$ Institute of Virology, Charité Universitätsmedizin Berlin, 10117 Berlin, Germany. ${ }^{7}$ Institute for Anatomy and Cell Biology, Ulm University, 89081 Ulm Germany. ${ }^{8}$ Institute of Medical Microbiology and Hygiene, Ulm University Medical Centre, 89081 Ulm, Germany. ${ }^{9}$ Institute of Medical Science, The University of Tokyo, 1088639 Tokyo, Japan. $\underline{\text { konstantin.sparrer@uni-ulm.de or Frank.Kirchhoff@uni-ulm.de }}$

Running title: IFITMs promote SARS-CoV-2 infection

KEYWORDS: SARS-CoV-2, Interferon-induced transmembrane proteins, spike glycoproteins, human lung cells, viral entry cofactors 
29 Interferon-induced transmembrane proteins (IFITMs 1, 2 and 3) are thought to restrict

30 numerous viral pathogens including severe acute respiratory syndrome coronaviruses (SARS-

$31 \mathrm{CoVs}$ ). However, most evidence comes from single-round pseudovirus infection studies of

32 cells that overexpress IFITMs. Here, we verified that artificial overexpression of IFITMs

33 blocks SARS-CoV-2 infection. Strikingly, however, endogenous IFITM expression was

34 essential for efficient infection of genuine SARS-CoV-2 in human lung cells. Our results

35 indicate that the SARS-CoV-2 Spike protein interacts with IFITMs and hijacks them for

36 efficient viral entry. IFITM proteins were expressed and further induced by interferons in

37 human lung, gut, heart and brain cells. Intriguingly, IFITM-derived peptides and targeting

38 antibodies inhibited SARS-CoV-2 entry and replication in human lung cells, cardiomyocytes

39 and gut organoids. Our results show that IFITM proteins are important cofactors for SARS-

40 CoV-2 infection of human cell types representing in vivo targets for viral transmission,

41 dissemination and pathogenesis and suitable targets for therapeutic approaches. 


\section{INTRODUCTION}

43 SARS-CoV-2 is the cause of pandemic Coronavirus disease 2019 (COVID-19). Originating

44 from China in late 2019, the virus has infected more than 76 million people around the globe

45 (https://coronavirus.jhu.edu/map.html). While SARS-CoV-2 spreads more efficiently than

46 SARS-CoV and MERS-CoV, the previously emerging causative agents of severe acute

47 respiratory syndromes (SARS), it shows a lower case-fatality rate ( $\sim 2$ to $5 \%$ ), compared to

$48 \sim 10 \%$ and almost $40 \%$, respectively ${ }^{1-3}$. The reasons for this efficient spread and the

49 mechanisms underlying the development of severe COVID-19 are incompletely understood

50 but the ability of SARS-CoV-2 to evade or counteract innate immune mechanisms may play a

51 key role ${ }^{4}$.

52 Here, we focused on innate immune effectors that are thought to target the first essential step 53 of SARS-CoV-2 replication: entry into its target cells. A prominent family of interferon (IFN)

54 stimulated genes (ISGs) known to inhibit fusion between the viral and cellular membranes are

55 interferon-inducible transmembrane (IFITM) proteins ${ }^{5,6}$. The three best characterised members

56 of the IFITM family are IFITM1, IFITM2 and IFITM $3^{7-10}$. They contain different sorting

57 motifs and IFITM1 is mainly localised at the plasma membrane, while IFITM2 and 3 are found

58 inside the cell on endo-lysosomal membranes ${ }^{7}$. Thus, IFITM proteins may act at different sites

59 of viral entry and it has been reported that they restrict multiple classes of enveloped viral

60 pathogens including Influenza A viruses, Flaviviruses, Rhabdoviruses, Bunyaviruses and

61 human immunodeficiency viruses ${ }^{6,11}$. The molecular mechanism(s) underlying the antiviral

62 activity of IFITMs are not fully understood. However, recent reports suggest that they modulate

63 membrane rigidity and curvature to prevent fusion of the viral and cellular membranes ${ }^{12-14}$.

64 It has also been reported that IFITM proteins inhibit human coronaviruses including SARS-

$65 \mathrm{CoV}-1$ and SARS-CoV-2 as well as MERS-CoV ${ }^{11,15}$. However, most results were obtained

66 using Spike containing viral pseudoparticles and cell lines overexpressing the IFITM proteins

67 and frequently also the viral ACE2 receptor. Here, we confirmed and expanded previous results 
showing that IFITM proteins block SARS-CoV-2 entry under such artificial experimental conditions. In striking contrast, however, endogenous IFTIM proteins were essential for efficient infection and replication of genuine SARS-CoV-2 in various types of human cells.

We found that IFITM proteins are expressed in human cell types involved in virus transmission, dissemination to various organs, and development of severe COVID-19. In further support of an important role of IFITM proteins as entry cofactors of SARS-CoV-2, IFITM-derived peptides and targeting antibodies efficiently inhibited SARS-CoV-2 infection of human lung, heart and gut cells. Our unexpected finding that SARS-CoV-2 hijacks human IFITM proteins for efficient infection helps to explain the rapid spread of this pandemic viral pathogen.

\section{Results}

Overexpressed IFITMs block and endogenous IFITMs boost SARS-CoV-2 infection. It

has been reported that overexpression of IFITM proteins prevents entry of viral particles pseudotyped with the Spike (S) proteins of SARS- and MERS-CoVs ${ }^{9,11,15}$. In agreement with these previous findings, we found that IFITM1, IFITM2 and (less efficiently) IFITM3 dosedependently inhibited SARS-CoV-2 S-mediated entry of Vesicular-Stomatitis-Virus pseudoparticles (VSVpp) into transfected HEK293T cells (Fig. 1a, Extended Data Fig. 1a, b). Inhibition of SARS-CoV-2 S-mediated infection by IFITM proteins was confirmed using lentiviral pseudoparticles (LVpp, Extended Data Fig. 1c). In contrast, IFITMs did not significantly affect VSV-G-dependent entry (Extended Data Fig. 1d). To examine the impact of endogenous IFITM expression on S-mediated VSVpp infection, we performed siRNA knock-down (KD) studies in the human epithelial lung cancer cell line Calu-3, which expresses ACE $2^{16}$ and increased levels of all three IFITM proteins upon IFN treatment (Extended Data Fig. 2a). On average, silencing of IFITM expression (Extended Data Fig. 2b) enhanced VSVpp infection mediated by SARS-CoV S proteins about 3- to 7-fold (Fig. 1b). To determine whether overexpression of IFITMs also affects genuine SARS-CoV-2 replication, we infected 
HEK293T cells overexpressing ACE2 alone or together with individual IFITM proteins. In agreement with the inhibitory effects on S containing VSVpp and LVpp, IFITM1 and IFITM2 prevented viral RNA production almost entirely, while IFITM3 achieved $\sim 5$-fold inhibition (Fig. 1c).

To approximate the in vivo situation, we also examined the role of endogenous IFITM expression on genuine SARS-CoV-2 infection of human lung cells. In striking contrast to the results obtained with pseudovirions and/or IFITM overexpression, silencing of endogenous IFITM expression in Calu-3 cells strongly impaired viral RNA production (Fig. 1d, Extended Data Fig. 2c-e). On average, IFITM2 reduced viral RNA yields by $\sim 20$-fold in the absence and by $\sim 68$-fold in the presence of IFN- $\beta$. Consequently, the amount of infectious SARS-CoV-2 particles in the cell culture supernatant was reduced by several orders of magnitude upon silencing of IFITM2 and to a lesser extent also by depletion of IFITM1 and IFITM3 (Fig. 1e).

Titration analyses showed that IFITMs do not promote SARS-CoV-2 infection in transfected HEK239T cells over a broad range of expression levels (Extended Data Fig. 3). Thus, the opposing effects of transient and endogenous IFITM expression were not just due to different expression levels.

IFITMs enhance SARS-CoV-2 infection of primary human lung cells. To confirm that the requirement of endogenous IFITM expression for efficient SARS-CoV-2 replication is not limited to Calu-3 cells, we silenced IFITM proteins in primary small airway epithelial cells (SAEC) isolated from normal human lung tissues. Western blot analyses showed that SAEC cells express all three IFITM proteins and type I or II IFN treatment enhanced the expression levels $\sim 2-5$-fold (Fig. 2a). siRNA-mediated silencing strongly reduced the expression of IFITM proteins (Fig. 2b) and was associated with $\sim 40$ - to 50 -fold lower levels of SARS-CoV-2 RNA production in the presence of IFN- $\beta$ (Fig. 2c). Silencing of IFITM1 also clearly reduced viral RNA yields in the absence of IFN treatment (Fig. 2c). Altogether, IFITM1 was more critical 
120 for efficient SARS-CoV-2 replication in SAEC cells than in Calu-3 cells (Figs. 1d, 2c). It is

121 thought that IFITM1 is mainly found at the cell surface, while IFITM2 is preferentially

122 localized in early endosomes ${ }^{6,7}$. SARS-CoV-2 may enter cells at their surface as well as in

123 endosomes ${ }^{17}$. Thus, together with differences in the expression levels of specific IFITM

124 proteins, cell-type-dependent differences in the major sites of viral fusion may explain

125 differences in the relative dependency of SARS-CoV-2 on endogenous IFITM1 or IFITM2

126 expression. In contrast to the results obtained in Calu-3 cells (Extended Data Fig. 2e), IFN- $\beta$

127 enhanced rather than inhibited SARS-CoV-2 replication in SAEC cells (Fig. 2c). While this

128 finding came as surprise, it is reminiscent of previous data showing that IFN treatment

129 promotes infection by human coronavirus $\mathrm{HCoV}-\mathrm{OC} 43$. Notably, this $\mathrm{CoV}$ was proposed to

130 hijack IFITM3 for efficient entry ${ }^{18}$. Taken together, our results show that endogenous

131 expression of IFITM proteins promotes SARS-CoV-2 replication in primary human lung cells,

132 especially in the presence of IFN.

134 Endogenous IFITMs promote an early step of SARS-CoV-2 infection. To address the

mechanisms underlying these opposing effects of IFITMs, we examined the effect of IFITM proteins on SARS-CoV-2 S-mediated fusion under various conditions. To analyse the impact of IFITMs on S-mediated fusion between virions and target cells, we used HIV-1 particles containing $\beta$-lactamase-Vpr fusions as previously described ${ }^{19}$, except that the virions contained the SARS-CoV-2 S instead of the HIV-1 Env protein. In agreement with the documented role of IFITMs as inhibitors of viral fusion ${ }^{12,14}$, transient overexpression of all three IFITM proteins blocked fusion of SARS-CoV-2 S HIVpp ${ }^{19}$ with ACE2 expressing HEK293T cells (Extended Data Fig. 4a). Consistent with recent data $^{20}$, results from a split-GFP assay showed that artificial overexpression of IFITMs also inhibits HEK293T cell-to-cell fusion mediated by the

144 SARS-CoV-2 S protein and the ACE2 receptor (Extended Data Fig. 4b). To analyse the impact 145 of endogenous IFITM expression on genuine SARS-CoV-2 entry, we determined the levels of 
viral RNA in the cells at different time points after infection of Calu-3 cells. Already at $6 \mathrm{~h}$

post-infection, depletion of IFITMs 1, 2 and 3 reduced the levels of viral RNA in the cells about

overexpressed proteins, endogenous IFITM expression is required for efficient SARS-CoV-2 entry into human lung cells.

The SARS-CoV-2 Spike interacts with IFITM proteins. It is thought that the broadof SARS-CoV-2 to utilize IFITMs for efficient infection of human lung cells may instead involve specific interactions between the viral S protein and IFITMs, we performed proximity ligation assays (PLA; Extended Data Fig. 5) ${ }^{22}$. The result revealed higher number of foci for S and IFITM2 compared to IFITM1 and 3 in SARS-CoV-2 infected Calu-3 cells (Fig. 3b), for SARS-CoV-2 replication in this cell type (Fig. 2c), high levels of PLA signals were detected for S and IFITM1 in infected SAEC cells (Fig. 3b). Assessing integral membrane proteinprotein interactions using the mammalian-membrane two-hybrid (MaMTH) assay ${ }^{23}$ provided further evidence that SARS-CoV-2 S interacts with IFITM proteins (Fig. 2d, Extended Data

Fig. 6). Finally, the SARS-CoV-2 S-protein co-immunoprecipitated IFITM2 and, to a lesser extent, IFITM1 and IFITM3 (Fig. 2e). Altogether, several independent lines of evidence support that the S protein of SARS-CoV-2 interacts with human IFITM proteins. 
receptor. Knockdown of IFITM2 and, to a lesser extent, IFITM3 enhanced the number of S/ACE2 PLA foci after infection of Calu-3 cells with genuine SARS-CoV-2 (Fig. 4a). The number of S/ACE2 foci rapidly declined (Fig. 4b) and S/RAB5A signals strongly increased (Fig. 4c) after switching SARS-CoV-2 infected Calu-3 cell cultures from ice to $37^{\circ} \mathrm{C}$, most likely indicating S-mediated virion fusion in endosomes. The magnitude of these effects was reduced upon silencing of IFITM2 expression (Fig. 4d) and endogenous IFITM expression usually decreased the number of S molecules that are in close proximity to the ACE2 receptor. It is tempting to speculate that IFITMs reduce the number of S/ACE2 signals by accelerating virion fusion and hence the disappearance of signals. However, further studies are required to elucidate the details of the underlying mechanism(s).

IFITMs are targets for inhibition of SARS-CoV-2 replication. Our discovery that IFITMs serve as cofactors for efficient SARS-CoV-2 infection suggested that they might represent targets for viral inhibition. To address this, we examined the effect of antibodies targeting the N-terminal region of the three IFITM proteins (Fig. 5a) on SARS-CoV-2 infection of Calu-3 cells. Indeed, antibodies against the N-terminal region of IFITM2 or recognizing all three IFITM proteins inhibited SARS-CoV-2 replication in Calu-3 cells up to 50-fold, while antibodies against IFITM1 or IFITM3 had negligible inhibitory effects (Fig. 5b). Since the membrane topology of IFITMs proteins is under debate ${ }^{7}$, we verified by flow cytometry analyses that the N-terminal region of IFITMs is accessible to antibody binding (Extended Data Fig. 7). Further analyses showed that peptides corresponding to the N-proximal region of IFITM2 that is recognized by inhibitory antibodies also efficiently impair SARS-CoV-2 replication (Fig. 5c). In contrast, the corresponding IFITM3-derived peptide, which differs in four of the 23 residues from the IFITM2-derived peptide, and a scrambled control peptide of the same length and amino acid composition had little if any effect on viral RNA yields. Notably, incubation of SARS-CoV-2 virions with the peptides prior to infection had no 
inhibitory effect (Extended Data Fig. 8). Thus, similarly to other inhibitors of SARS-CoV-2 infection $^{24,25}$ the IFITM2-derived peptides might target a region in the viral S protein that only becomes accessible during the entry process.

\section{IFITM-derived peptides or targeting antibodies protect gut organoids and} cardiomyocytes against SARS-CoV-2. To better assess the potential relevance of IFITMs for viral spread and pathogenesis in SARS-CoV-2-infected individuals, we analysed their expression in various cell types. We found that IFITM proteins are efficiently expressed in primary human lung bronchial epithelial (NHBE) cells, neuronal cells, and intestinal organoids derived from pluripotent stem cells (Extended Data Fig. 9a-c). These cell types and organoids represent the sites of SARS-CoV-2 entry and subsequent spread, i.e. the lung and the gastrointestinal $\operatorname{tract}^{26-28}$, and the potential targets responsible for neurological manifestations of COVID-1929. Confocal microcopy analyses confirmed efficient induction of IFITM expression by IFN- $\beta$ (Fig. 6a). NHBE cells and cultures of neuronal cells did not support efficient SARS-CoV-2 replication precluding meaningful inhibition analyses. Gut organoids, however, are susceptible to SARS-CoV-2 replication ${ }^{27}$ and treatment with the IFITM2-derived peptide or an antibody targeting the N-terminus of IFITMs strongly reduced viral RNA production (Fig. 6b). Independent infection experiments confirmed that both agents significantly reduce viral $\mathrm{N}$ protein expression and cytopathic effects in gut organoids (Fig. 6c). Following up on recent evidence that SARS-CoV-2 causes cardiovascular disease ${ }^{30}$, we investigated viral replication in human iPSC-derived cardiomyocytes. In agreement with published data ${ }^{31}$, beating cardiomyocytes were highly susceptible to viral replication (Fig. 6d). All three IFITM proteins were expressed in cardiomyocytes and further induced by virus infection (Fig. 6e). On average, treatment of cardiomyocytes with the IFITM2- or 3-derived peptides reduced the efficiency of SARS-CoV-2 replication by $\sim 10$ - and 5-fold, respectively (Fig. 6f). In addition, treatment with these peptides suppressed or prevented disruptive effects 
224 of virus infection on the ability of cardiomyocytes to beat in culture. Thus, IFITMs can be

225 targeted to inhibit SARS-CoV-2 replication in cells from various human organs, including the

226 lung, gut and heart.

\section{Discussion}

229 The present study demonstrates that endogenous expression of IFITMs is required for efficient 230 replication of SARS-CoV-2 in human lung cells. In addition, we show that IFITMs can be targeted to inhibit SARS-CoV-2 infection of human lung, gut and heart cells. These findings came as surprise since IFITMs have been reported to inhibit SARS-CoV, MERS-CoV and, very recently, SARS-CoV-2 S-mediated infection ${ }^{11,15,32}$. Confirming and expanding these previous studies, we show that artificial overexpression of IFITM proteins in HEK293T cells prevents S-mediated VSVpp and HIVpp fusion as well as genuine SARS-CoV-2 entry. However, exactly the opposite was observed for genuine SARS-CoV-2 upon manipulation of endogenous IFITM expression in human lung cells: silencing of all three IFITM proteins reduced SARS-CoV-2 entry. Our results provide novel and highly unexpected insights into the role of IFITM proteins in the spread and pathogenesis of SARS-CoV-2 and suggest that these supposedly antiviral factors are hijacked by SARS-CoV-2 as cofactors for efficient entry.

While wildtype IFITM proteins have generally been described as inhibitors of SARS and MERS coronaviruses (Ref) specific point mutations may convert IFITM3 from an inhibitor to 243 an enhancer Spike-mediated pseudoparticle transduction ${ }^{33}$. It has been reported that overexpression of IFITM3 promotes infection by hCoV-OC43, one of the causative agents of common colds ${ }^{18}$. However, IFITM3 was least relevant for SARS-CoV-2 infection in the present study. Thus, although both human coronaviruses may highjack IFITMs for efficient

247 infection they show distinct preferences for specific IFITM proteins. It is under debate whether 248 SARS-CoV-2 mainly fuses at the cell surface or in endosomes and cell-type-specific 249 differences may explain why IFITM2 plays a key role in Calu-3 cells, while IFITM1 is at least 
as important in SAEC cells. Most importantly, our results clearly demonstrate that IFITM proteins act as critical cofactors for efficient SARS-CoV-2 infection under the most physiological conditions.

We currently do not yet understand why overexpressed and endogenous IFITM proteins have opposite effects on SARS-CoV-2 infection. However, artificial overexpression may change the topology, localisation and endocytic activity of proteins and it has been reported that specific mutations in IFITM3 affecting these features may convert IFITM3 from an inhibitor to an enhancer of coronavirus infection ${ }^{9,34}$. The antiviral activity of IFITMs is very broad and does not involve interactions with specific viral glycoproteins ${ }^{6,7}$. In contrast, the ability of SARS-CoV-2 to hijack IFITMs for efficient entry seems to involve specific interactions between the N-terminal region of IFITMs and the viral S protein (outlined in Extended Data Fig. 10).

IFITMs are strongly induced during the innate immune response in SARS-CoV-2-infected individuals ${ }^{35,36}$. Thus, utilization of IFITMs as infection cofactors may promote SARS-CoV-2 invasion of the lower respiratory tract as well as spread to secondary organs especially under inflammatory conditions. Further studies are required but efficient expression in neurons and cardiomyocytes suggest that IFITMs may play a role in the well documented neuronal and cardiovascular complications associated with SARS-CoV-2 infection (Ref). Perhaps most intriguingly, we show that IFITM-derived peptides and antibodies against the N-terminal region of IFITM2 efficiently inhibit SARS-CoV-2 replication. Targeting cellular IFITM proteins as a therapeutic approach should reduce the risk of viral resistance and be well tolerated since these factors are mainly known for their antiviral activity and may not exert critical physiological functions. 


\section{Methods}

277 Cell culture. All cells were cultured at $37^{\circ} \mathrm{C}$ in a $5 \% \mathrm{CO}_{2}$ atmosphere. Human embryonic 278 kidney 293T cells (HEK293T; ATCC) were maintained in Dulbecco's Modified Eagle 279 Medium (DMEM) supplemented with 10\% heat-inactivated fetal calf serum (FCS), L280 glutamine $(2 \mathrm{mM})$, streptomycin $(100 \mu \mathrm{g} / \mathrm{ml})$ and penicillin $(100 \mathrm{U} / \mathrm{ml})$. HEK293T were 281 provided and authenticated by the ATCC. Caco-2 (human epithelial colorectal 282 adenocarcinoma) cells were maintained in DMEM containing 10\% FCS, glutamine (2 mM), streptomycin $(100 \mu \mathrm{g} / \mathrm{ml})$ and penicillin $(100 \mathrm{U} / \mathrm{ml})$, NEAA supplement (Non-essential amino acids (1 mM)), sodium pyruvate (1 mM). Calu-3 (human epithelial lung adenocarcinoma) cells were cultured in Minimum Essential Medium Eagle (MEM) supplemented with $10 \%$ FCS (during viral infection) or $20 \%$ (during all other times), penicillin $(100 \mathrm{U} / \mathrm{ml})$, streptomycin $(100 \mu \mathrm{g} / \mathrm{ml})$, sodium pyruvate $(1 \mathrm{mM})$, and NEAA supplement $(1 \mathrm{mM})$. Hybridoma cells (Mouse I1 Hybridoma CRL-2700; ATCC) were cultured in Roswell Park Memorial Institute (RPMI) 1640 medium supplemented with 10\% FCS, L-glutamine (2 mM), streptomycin (100 $\mu \mathrm{g} / \mathrm{ml})$ and penicillin $(100 \mathrm{U} / \mathrm{ml})$. Vero cells (ATCC, CCL-81) cells were maintained in

DMEM containing 2.5\% FCS, glutamine $(2 \mathrm{mM})$, streptomycin $(100 \mu \mathrm{g} / \mathrm{ml})$ and penicillin $(100 \mathrm{U} / \mathrm{ml})$, NEAA supplement (Non-essential amino acids $(1 \mathrm{mM}))$, sodium pyruvate $(1 \mathrm{mM})$. Monoclonal anti-VSV-G containing supernatant was aliquoted and stored at $-20^{\circ} \mathrm{C}$. NHBE (primary human bronchial/tracheal epithelial, Lonza) cells were grown in Bronchial Epithelial Cell Growth Basal Medium (BEGM, Lonza) and Bronchial Epithelial Cell Growth Medium SingleQuots Supplements and Growth Factors (Lonza). SAEC (Small Airway Epithelial cells, Lonza) were grown in Small Airway Epithelial Cell Growth Basal Medium (SABM, Lonza) 
301 Human hESC cultivation and gut organoids differentiation. Human embryonic stem cell 302 (hESC) line HUES8 (Harvard University) was used with permission from the Robert Koch 303 Institute according to the "Approval according to the stem cell law" AZ 3.04.02/0084. Cells 304 were cultured on hESC Matrigel (Corning) in mTeSR1 medium (Stemcell Technologies) at 5\% $305 \mathrm{CO}_{2}$ and $37^{\circ} \mathrm{C}$. Medium was changed every day and cells were splitted twice a week with 306 TrypLE Express (Invitrogen). Experiments involving human stem cells were approved by the 307 Robert-Koch-Institute (Approval according to the stem cell law 29.04.2020).

308 Cardiomyocyte differentiation. Human episomal hiPSCs (\#A18945, Thermo Fisher 309 Scientific) at passage 2 were split using TrypLE (\#12604-013, Thermo Fisher Scientific) to 310 generate a single cell suspension. $18000 \mathrm{iPS}$ cells were seeded on Geltrex (\#A1413302, Thermo 311 Fisher Scientific) matrix coated 12 well plates. 3 days post splitting differentiation protocol 312 into iPS cardiomyocytes using the PSC cardiomyocytes Differentiation Kit (\#A29212-01, 313 Thermo) was initiated. Contracting iPSC-derived cardiomyocytes were present 14 days post 314 differentiation initiation.

315 Neuronal differentiation. Human iPSC, either generated from keratinocytes as previously described $^{37}$ or commercially purchased from the iPSC Core facility of Cedars Sinai (Los Angeles, California), were cultured at $37^{\circ} \mathrm{C}\left(5 \% \mathrm{CO}_{2}, 5 \% \mathrm{O}_{2}\right)$ on Matrigel-coated (Corning, 354277) 6-well plates using mTeSR1 medium (Stem Cell Technologies, 83850). Neuronal differentiation was chemically induced by culturing hiPSC colonies in suspension in ultra-low attachment T75 flasks (Corning, 3815), to allow the formation of embryoid bodies (EBs). During the first 3 days of differentiation, cells were cultivated in DMEM/F12 (Gibco, 31331028) containing 20\% knockout serum replacement (Gibco, 10828028), 1\% NEAA, 1\% $\beta$ mercaptoethanol, 1\% antibiotic-antimycotic, SB-431542 $10 \mu \mathrm{M}$ (Stemcell Technologies, 72232), Dorsomorphin $1 \mu \mathrm{M}$ (Tocris, 3093), CHIR $990213 \mu \mathrm{M}$ (Stemcell Technologies, 72054), Pumorphamine $1 \mu \mathrm{M}$ (Miltenyi Biotec, 130-104-465), Ascorbic Acid 200ng/ $\mu \mathrm{L}$, 
$3270.5 \% \mathrm{~N} 2$ supplement (Gibco, 17502-284). From the fourth day on, medium was switched to 328 DMEM/F12 added with 24 nM sodium selenite (Sigma-Aldrich, S5261), 16 nM progesterone 329 (Sigma-Aldrich, P8783), $0.08 \mathrm{mg} / \mathrm{mL}$ apotransferrin (Sigma-Aldrich, T2036), $0.02 \mathrm{mg} / \mathrm{mL}$, 330 Insulin (Sigma-Aldrich, 91077C), $7.72 \mu \mathrm{g} / \mathrm{mL}$ putrescine (Sigma-Aldrich, P7505), 1\%NEAA, $3311 \%$ antibiotic-antimycotic, $50 \mathrm{mg} / \mathrm{mL}$ heparin (Sigma-Aldrich, H4783), $10 \mu \mathrm{g} / \mathrm{mL}$ of the 332 neurotrophic factors BDNF (Peprotech, 450-02), GDNF (Peprotech, 450-10), and IGF1 333 (Peprotech, 100-11), $10 \mu \mathrm{M}$ SB-431542, $1 \mu \mathrm{M}$ dorsomorphin, $3 \mu \mathrm{M}$ CHIR 99021, $1 \mu \mathrm{M}$ 334 pumorphamine, $150 \mu \mathrm{M}$. vitamin $\mathrm{C}, 1 \mu \mathrm{M}$ retinoic acid, $500 \mu \mathrm{M}$ cAMP, $1 \%$ Neurocult 335 supplement, $0.5 \% \mathrm{~N} 2$ supplement. After 5 further days, neurons were dissociated to single cell 336 suspension and plated onto $\mu$ Dishes, or 6-well plates (Corning, 353046) pre-coated with 337 Growth Factor Reduced Matrigel (Corning, 356231).

Expression constructs. Expression plasmids encoding for IFITM1, IFITM2 and IFITM3 (pCG_IFITM1, pCG_IFITM2, pCG_IFITM3 and pCG_IFITM1-IRES_eGFP, pCG_IFITM2IRES_eGFP and pCG_IFITM3-IRES_BFP) were PCR amplified and subcloned in pCG based backbones using flanking restriction sites XbaI and MluI. pCG_SARS-CoV-2-SpikeIRES_eGFP (humanized), encoding the spike protein of SARS-CoV-2 isolate Wuhan-Hu-1, NCBI reference Sequence YP_009724390.1 while pCG_SARS-CoV-2-Spike C-V5IRES_eGFP was PCR amplified and subcloned using XbaI+MluI, while pCG_SARS-CoV2Spike C-V5-IRES_eGFP was PCR amplified and subcloned using XbaI+MluI. To generate the pLV-EF1a-human ACE2-IRES-puro, pTargeT-hACE2 was provided by Sota Fukushi and Masayuki Saijo (National Institute of Infectious Diseases, Tokyo, Japan). The ORF of ACE2 was extracted with MluI and SmaI and then inserted into the MluI-HpaI site of pLV-EF1aIRES-Puro.

Pseudoparticle stock production. To produce pseudotyped VSV(luc/GFP) $\Delta \mathrm{G}$ particles, HEK293T cells were transfected with pCG_SARS-CoV-2-Spike C-V5-IRES_GFP, as 352 previously described ${ }^{38}$. 24 hours post transfection, the cells were infected with 
353 VSV $\Delta \mathrm{G}(\mathrm{GFP} / \mathrm{luc}) * \mathrm{VSV}-\mathrm{G}$ at an MOI of 1 . The inoculum was removed after $1 \mathrm{~h}$. Pseudotyped 354 particles were harvested at $16 \mathrm{~h}$ post infection. Cell debris was removed by centrifugation at $3552000 \mathrm{rpm}$ for $5 \mathrm{~min}$. Residual input particles carrying VSV-G were blocked by adding $10 \%$ 356 (v/v) of I1 Hybridoma supernatant (I1, mouse hybridoma supernatant from CRL-2700; ATCC) 357 to the cell culture supernatant. To produce pseudotyped HIV-1(fLuc) $\Delta e n v$ particles, HEK293T 358 cells were transfected with pCMVdR8.91 (Addgene) and pSEW-luc2 (Promega, \# 9PIE665 ) 359 or pCMV4-BlaM-vpr (Addgene, \#21950) as well as pCG_SARS-CoV-2-Spike C-V5360 IRES_eGFP using TransIT-LT1 according to the manufacturer's protocol. Six hours post 361 transfection, the medium was replaced with DMEM containing only $2.5 \%$ FCS. The particles 362 were harvested 48 hours post transfection. Cell debris was pelleted by centrifugation at $2000 \mathrm{rpm}$ for $5 \mathrm{~min}$.

Target cell assay. HEK293T cells were transiently transfected using PEI ${ }^{38}$ with pLV-EF1ahuman ACE2-IRES-puro and pCG-IFITM1-IRES_eGFP or pCG-IFITM2-IRES_eGFP or pCG-IFITM3-IRES_BFP. $24 \mathrm{~h}$ post transfection, cells were transduced/infected with HIV-1Aenv(fLuc)* SARS-CoV-2 S or VSV(luc) $\Delta \mathrm{G} * \mathrm{SARS}-\mathrm{CoV}-2 \mathrm{~S}$ particles. $16 \mathrm{~h}$ post infection Luciferase activity was quantified.

Luciferase assay. To determine viral gene expression, the cells were lysed in $300 \mu$ of was determined using the Luciferase Assay Kit (Luciferase Cell Culture, Promega) according to the manufacturer's instructions on an Orion microplate luminometer (Berthold).

373 Vpr-BlaM fusion assay. HEK293T cells were seeded and transiently transfected using PEI ${ }^{38}$ with pLV-EF1a-human_ACE2-IRES-puro and pCG_IFITM1, pCG_IFITM2 or pCG_IFITM3. 24 hours post transfection, cells were transferred to a 96-well plate. On the next day, cells were infected with $50 \mu \mathrm{l} \mathrm{HIV-1} \Delta$ env (BlaM-Vpr)-*SARS-CoV-2-S particles for $2.5 \mathrm{~h}$ at $37{ }^{\circ} \mathrm{C}$, followed by washing with PBS. Cells were detached and stained with CCF2/AM (1 mM) as 
378 previously described ${ }^{39}$. Finally, cells were washed and fixed with $4 \%$ PFA. The change in

379 emission fluorescence of CCF2 after cleavage by the BlaM-Vpr chimera was monitored by

380 flow cytometry using a FACSCanto II (BD).

SARS-CoV-2 virus stock production. BetaCoV/Netherlands/01/NL/2020 or BetaCoV/

France/IDF0372/2020 was propagated on Vero E6 infected at an MOI of 0.003 in serum-free

medium containing $1 \mu \mathrm{g} / \mathrm{ml}$ trypsin as previously described ${ }^{16}$. Briefly, the cells were inoculated

for $2 \mathrm{~h}$ at $37^{\circ} \mathrm{C}$ before the inoculum was removed. The supernatant was harvested $48 \mathrm{~h}$ post

infection upon visible cytopathic effect (CPE). To remove the debris, the supernatants were

centrifuged for $5 \mathrm{~min}$ at $1,000 \times \mathrm{g}$, then aliquoted and stored at $-80^{\circ} \mathrm{C}$. Infectious virus titre

was determined as plaque forming units (PFU).

Plaque-forming Unit Assay. The plaque-forming unit (PFU) assay was performed as

previously described ${ }^{16}$. SARS-CoV-2 stocks were serially diluted and confluent monolayers of

Vero E6 cells infected. After incubation for $2 \mathrm{~h}$ at $37^{\circ} \mathrm{C}$ with shaking every $20 \mathrm{~min}$. The cells were overlaid with $1.5 \mathrm{ml}$ of $0.8 \%$ Avicel RC-581 (FMC) in medium and incubated for 3 days.

Cells were fixed with $4 \%$ PFA at room temperature for $45 \mathrm{~min}$. After the cells were washed with PBS once $0.5 \mathrm{ml}$ of staining solution $(0.5 \%$ crystal violet and $0.1 \%$ triton in water $)$. After

$20 \mathrm{~min}$ incubation at room temperature, the staining solution was removed using water, virusinduced plaque formation quantified, and PFU per ml calculated.

qRT-PCR. N (nucleoprotein) RNA levels were determined in supernatants or cells collected from SARS-CoV-2 infected cells $6 \mathrm{~h}, 24 \mathrm{~h}$ or $48 \mathrm{~h}$ post-infection. Total RNA was isolated using the Viral RNA Mini Kit (Qiagen) according to the manufacturer's instructions. qRTPCR was performed according to the manufacturer's instructions using TaqMan Fast Virus 1- 
obtain viral copy numbers. All reactions were run in duplicates. (Forward primer (HKU-NF): 5'-TAA TCA GAC AAG GAA CTG ATT A-3'; Reverse primer (HKU-NR): 5'-CGA AGG TGT GAC TTC CAT G-3'; Probe (HKU-NP): 5'-FAM-GCA AAT TGT GCA ATT TGC GGTAMRA). GAPDH primer/probe sets (Thermo Fisher) were used for normalization of cellular RNA levels.

IFITM1, 2 and 3 knock-down. $24 \mathrm{~h}$ and $96 \mathrm{~h}$ after seeding, Calu-3 or SAEC cells were transfected twice with $20 \mu \mathrm{M}$ of either non-targeting siRNA or IFITM1, IFITM2 or IFITM3 specific siRNA using Lipofectamine RNAiMAX (Thermo Fisher) according to the manufacturer's instructions. $14 \mathrm{~h}$ post transfection, medium was replaced with fresh medium supplemented with $500 \mathrm{U} / \mathrm{ml} \mathrm{IFN- \beta}$ in the indicated conditions. $7 \mathrm{~h}$ after the second transfection, Calu-3 or SAEC cells were infected with SARS-CoV-2 with an MOI of 0.05 and 2.5 respectively. $6 \mathrm{~h}$ later, the inoculum was removed, cells were washed once with PBS and supplemented with fresh media. $48 \mathrm{~h}$ post infection, cells and supernatants were harvested for Western blot and qRT-PCR analysis respectively.

Stimulation with type I interferon. Calu-3, NHBE cells and SAEC were seeded in 12-well plates. For the gut organoids stimulation, HUES88 were seeded in 24-well-plates were coated with growth factor reduced (GFR) Matrigel (Corning) and in mTeSR1 with $10 \mu \mathrm{M}$ Y-27632 (Stemcell technologies). The next day, differentiation to organoids was started at $80-90 \%$ confluency as previously described ${ }^{26}$. Cells or organoids were stimulated with IFN- $\alpha 2$ (500 U/ml, R\&D systems 11100-1), IFN- $\beta$ (500 U/ml, R\&D systems 8499-IF-010) or IFN- $\gamma(200$ U/ml, R\&D systems 285-IF-100). 3 days post-stimulation whole cell lysates were generated.

Cardiomyocytes infection and kinetics. Human iPSC-derived cardiomyocytes were cultures in 12 wells plates, until they were 3 to 4 weeks old and homogenously beating. Cells were infected with increasing MOIs $(0.1,0.25,0.5,1,2)$ of the BetaCoV/Netherlands/01/NL/2020 strain. $6 \mathrm{~h}$ post infection, cells were washed once with PBS to remove input virus and 
supplemented with fresh media. Virus-containing supernatant was harvested every day and replaced with fresh media until day 7 (as indicated). N gene RNA copies were determined by qRT-PCR and cells were harvested for Western blot analysis at the latest timepoint.

Peptides synthesis. The IFITM-derived peptides were synthetized by UPEP, Ulm using F-moc chemistry. Purification to homogeneity of more than $95 \%$ was done by reverse phase HPLC. Peptide stock were prepared in distilled water to a final concentration of $10 \mathrm{mg} / \mathrm{ml}$.

Inibition by IFITM antibodies and peptides. Calu-3 cells were seeded in 48 -well format (peptides assays), or in 24-well format (antibodies assay), 24h later cells were treated with increasing concentrations (20 and $80 \mu \mathrm{g} / \mathrm{ml}$ ) of IFITMs derived peptides (human IFITM2 long: EEQEVAMLGVPHNPAPPMSTVIH, human IFITM2 short: QEVAMLGVPHNAPPMSTVIH, mouse IFITM2 long: EEYGVTELGEPSNSAVVRTTVIN, human IFITM3 long: EEHEVAVLGAPHNPAPPTSTVIH， scrambled IFITM2: EGESGVTTATVEVVIERNNLPY) or blocking antibodies (15 and $30 \mu \mathrm{g} / \mathrm{ml})(\alpha$-ACE2 AK (AC18Z), Santa Cruz Biotechnology sc-73668; $\alpha$-IFITM1 Cell Signaling 13126 S, $\alpha$-IFITM2 Cell Signaling 13530S, $\alpha$-IFITM3 Proteintech 11714-1-AP, $\alpha$-IFITM1/2/3 (F-12) Santa Cruz Biotechnology sc374026) as indicated. 2 h post-treatment, cells were infected with SARS-CoV-2 with an MOI of 0.05. $6 \mathrm{~h}$ post-infection, cells were washed once with PBS and supplemented with fresh MEM medium. $48 \mathrm{~h}$ post-infection supernatants were harvested for qRT-PCR analysis. Cardiomyocytes were seeded in 12 -well plates, and treated with $100 \mu \mathrm{g} / \mathrm{ml}$ of indicated peptides $1 \mathrm{~h}$ prior to infection (MOI 0.01). $6 \mathrm{~h}$ post infection, cells were washed once with PBS to remove input virus and supplemented with fresh media. Virus-containing supernatant was harvested every day, replaced with fresh media until day 3, and fresh peptides $(100 \mu \mathrm{g} / \mathrm{ml})$ (as indicated). N gene RNA copies were determined by qRT-PCR. Gut organoids were treated with increasing concentrations (15 and $30 \mu \mathrm{g} / \mathrm{ml}$ ) of IFITMs derived peptides (mouse IFITM2 antibody blocking peptide Santa Cruz sc-373676 P) and blocking antibodies ( $\alpha$-ACE2 AK (AC18Z), Santa Cruz Biotechnology sc-73668, $\alpha$-IFITM1/2/3 (F-12) Santa Cruz 
454 Biotechnology sc-374026) as indicated. 1h30 post-treatment, organoids were infected with

455 SARS-CoV-2 with an MOI 0.15 as previously described ${ }^{40} .48 \mathrm{~h}$ post-infection gut organoids

456 were harvested for qRT-PCR analysis.

457 Virus treatment. Calu-3 cells were seeded in 48-wells, 24 h later SARS-COV-2 (0.05 MOI)

458 was incubated for $30 \mathrm{~min}$ at $37^{\circ} \mathrm{C}$ with indicated concentrations of IFITM-derived peptides. 50

$459 \mu 1$ of the inoculum were used to infect the cells. 6h later cells were supplemented with fresh

460 medium. $48 \mathrm{~h}$ post-infection supernatants were harvested for qRT-PCR analysis.

461 Flow cytometry analysis of IFITMs. HEK293T cells were transfected with pCG_IFITM1, 2

462 or 3 using PEI as previously described. Calu-3 cells were seeded $24 \mathrm{~h}$ before harvest in a 6 well

463 format. 24h post transfection and post seeding, cells were harvested using a scraper and stained

464 with the eBioscience Fixable Viability Dye eFluor 780 (Thermo Fisher) for 15 minutes at room

465 temperature in the dark. Afterwards cells were washed three times with PBS and fixed with

$466100 \mu 1$ of Reagent A (FIX \& PERM Fixation and Permeabilization Kit, Nordic MUbio) for 30

467 minutes at room temperature, washed three time with PBS and stained with primary antibody

468 ( $\alpha$-IFITM1 Cell Signaling 13126 S, $\alpha$-IFITM2 Cell Signaling 13530S, $\alpha$-IFITM3 Proteintech

469 11714-1-AP, $\alpha$-IFITM1/2/3 (F-12) Santa Cruz Biotechnology sc-374026, ) diluted 1:20 in PBS

470 or in Reagent B (FIX \& PERM Fixation and Permeabilization Kit Nordic MUbio) for $1 \mathrm{~h}$ at

$471 \quad 4^{\circ} \mathrm{C}$. Cells were washed three times with PBS and stained with secondary antibody (Goat Anti-

472 Rabbit IgG H\&L (PE), ab72465, Donkey Anti-Mouse IgG H\&L (PE) ab7003, 1:50) for $1 \mathrm{~h}$ at

$4734{ }^{\circ} \mathrm{C}$. After several washing with PBS, cells were resuspended in $100 \mu$ l of PBS.

474 Immunofluorescence of gut organoids. For histological examination, organoids were fixed

475 in $4 \%$ PFA over night at $4^{\circ} \mathrm{C}$, washed with PBS, and pre-embedded in $2 \%$ agarose (Sigma) in

476 PBS. After serial dehydration, intestinal organoids were embedded in paraffin, sectioned at 4

$477 \mu \mathrm{m}$, deparaffinized, rehydrated and subjected to heat mediated antigen retrieval in tris Buffer

$478(\mathrm{pH}$ 9) or citrate buffer ( $\mathrm{pH}$ 6). Sections were permeabilized with $0.5 \%$ Triton-X for 30 min at 
479 RT and stained over night with primary antibodies (rabbit anti-IFITM1 Cell Signaling 13126

480 S, 1:500 or rabbit anti-IFITM2 Cell Signaling \#13530S, 1:500 or rabbit anti-IFITM3 Cell

481 Signaling \#59212S, 1:250 or anti-SARS-CoV-2 N 1:500 or anti-E-Cadherin 1:500) diluted in

482 antibody diluent (Zytomed) in a wet chamber at $4^{\circ} \mathrm{C}$. After washing with PBS-Tween 20, slides

483 were incubated with secondary antibodies (Alexa Fluor IgG H+L, Invitrogen, 1:500) and 500

$484 \mathrm{ng} / \mathrm{ml}$ DAPI in Antibody Diluent for $90 \mathrm{~min}$ in a wet chamber at RT. After washing with PBS-

485 T and water, slides were mounted with Fluoromount-G (Southern Biotech). Negative controls

486 were performed using IgG controls or irrelevant polyclonal serum for polyclonal antibodies,

487 respectively. Cell borders were visualized by E-cadherin staining. Images were acquired using

488 a LSM 710 system.

GFP Split fusion assay. GFP1-10 and GFP11-expressing HEK293T cells were seeded 490 separately in a 24-well plate. One day post seeding, cells were transiently transfected using the 491 calcium-phosphate precipitation method ${ }^{41}$. GFP1-10 cells were co-transfected with increasing amounts $(0,8,16,32,64,125,250,500 \mathrm{ng})$ of pCG_IFTM1,pCG_IFITM2,pCG_IFITM3 and $250 \mathrm{ng}$ of pLV-EF1a-human ACE2-IRES-puro. GFP11 cells were transfected with $250 \mathrm{ng}$ of pCG_SARS-CoV-2-Spike C-V5 codon optimised. $16 \mathrm{~h}$ post transfection, GFP1-10 and GFP11 cells were co-cultured in poly-L-lysine-coated 24 -well plate. $24 \mathrm{~h}$ post co-culturing, cells were fixed with $4 \%$ PFA and cell nuclei were stained using NucRed Live 647 ReadyProbes Reagent 497 (Invitrogen) according to the manufacturer's instructions. Fluorescence imaging of GFP and 498 NucRed was performed using a Cytation3 imaging reader (BioTek Instruments). 12 images per well were recorded automatically using the NucRed signal for autofocusing. The GFP area was quantified using ImageJ.

Whole cell lysates. To determine expression of cellular and viral proteins, cells were washed in PBS and subsequently lysed in Western blot lysis buffer (150 mM NaCl, $50 \mathrm{mM}$ HEPES, 5 mM EDTA, $0.1 \%$ NP40, $\left.500 \mu \mathrm{M} \mathrm{Na}_{3} \mathrm{VO}_{4}, 500 \mu \mathrm{M} \mathrm{NaF}, \mathrm{pH} 7.5\right)$ supplemented with protease inhibitor (1:500, Roche) as previously described ${ }^{38}$. After 5 min of incubation on ice, samples 
505 were centrifuged $\left(4^{\circ} \mathrm{C}, 20 \mathrm{~min}, 14.000 \mathrm{rpm}\right)$ to remove cell debris. The supernatant was

506 transferred to a fresh tube, the protein concentration was measured and adjusted using Western

507 blot lysis buffer. Lysates from iPSC-derived neurons were prepared following previously

508 published protocols ${ }^{42}$. Briefly, neurons were harvested in cold PBS (Gibco) and centrifuged at

5095000 RPM for 3 minutes. Pellets were then resuspended and incubated at $4^{\circ} \mathrm{C}$ on an orbital

510 shaker for 2 hours in RIPA buffer. Lysate were then sonicated and protein concentration was

511 determined by Bradford assay.

512 SDS-PAGE and Immunoblotting. Western blotting was performed as previously described ${ }^{38}$.

513 In brief, whole cell lysates were mixed with 4x or 6x Protein Sample Loading Buffer (LI-COR,

514 at a final dilution of $1 \mathrm{x}$ ) supplemented with $10 \% \beta$-mercaptoethanol (Sigma Aldrich), heated

515 at $95^{\circ} \mathrm{C}$ for $5 \mathrm{~min}$, separated on NuPAGE $4 \pm 12 \%$ Bis-Tris Gels (Invitrogen) for 90 minutes at

$516100 \mathrm{~V}$ and blotted onto Immobilon-FL PVDF membranes (Merck Millipore). The transfer was

517 performed at a constant voltage of $30 \mathrm{~V}$ for 30 minutes. After the transfer, the membrane was

518 blocked in $1 \%$ Casein in PBS (Thermo Scientific). Proteins were stained using primary

519 antibodies against IFITM1 ( $\alpha$-IFITM1, Cell Signaling \#13126 S, 1:1000,), IFITM2 ( $\alpha$-IFITM2

520 Cell Signaling \#13530S, 1:1000), IFITM3 ( $\alpha$-IFITM3 Cell Signaling \#59212S, 1:1000) SARS

521 Spike CoV-2 (SARS-CoV-1/-2 (COVID-19) spike antibody [1A9], GTX-GTX632604,

522 1:1000), VSV-M (Mouse Monoclonal Anti-VSV-M Absolute antibody, ABAAb01404-21.0,

523 1:1000), actin (Anti-beta Actin antibody Abcam, ab8227, 1:5000 Abcam,), ACE2 (Rabbit

524 policclonal anti-ACE2 Abcam, ab166755, 1:1000) and Infrared Dye labelled secondary

525 antibodies (LI-COR IRDye). Membranes were scanned using LI-COR and band intensities

526 were quantified using Image Studio (LI-COR).

527 Proximity Ligation Assay. The proximity ligation assay (PLA) was performed as previously

528 described ${ }^{43}$. In brief, Calu-3 or SAEC were seeded in a 24-well plate on a cover slip glass. $24 \mathrm{~h}$

529 and $72 \mathrm{~h}$ post seeding, the cells were transfected with $20 \mu \mathrm{M}$ either non-targeting siRNA or

530 IFITM1 or IFITM3 siRNAs using RNAimax according to the manufacturer's instructions. 
531 Prior infection, cells were pre-chilled for 30 minutes at $4{ }^{\circ} \mathrm{C}$ and then infected with

532 VSV(luc) $\Delta G *$-SARS-CoV-2 S (MOI 2) or BetaCoV/France/IDF0372/2020 (MOI 0.05) for 2

$533 \mathrm{~h}$ on ice. Cells have been washed once with cold PBS and fixed with 4\% PFA. For staining

534 following antibodies were used: IFITM1 ( $\alpha$-IFITM1 Cell Signaling 13126 S), IFITM2 ( $\alpha$ -

535 IFITM2 Abcam 236735), IFITM3 ( $\alpha$-IFITM3 Cell Signaling 59212S), SARS Spike CoV-2

536 (SARS-CoV / SARS-CoV-2 (COVID-19) spike antibody [1A9], GTX-GTX632604), Rab5

537 alpha (Rab5 (RAB5A) Goat Polyclonal Antibody Origene AB0009-200) and ACE2 (Rabbit

538 polyclonal anti-ACE2 Abcam, ab166755). All in a concentration 1:100. Images were acquired

539 on a Zeiss LSM 710 and processed using ImageJ (Fiji).

540 Co-immunoprecipitation SARS-CoV-2 Spike and IFITMs. HEK293Ts were transfected

541 using PEI with $0.5 \mu \mathrm{g}$ pCG-SARS CoV2 Spike-V5 and $0.5 \mu \mathrm{g}$ of pCG IFITM1, IFITM2 or

542 IFITM3. $24 \mathrm{~h}$ later, samples were lysed with IP lysis buffer $(50 \mathrm{mM}$, Tris $\mathrm{pH} 8,150 \mathrm{mM} \mathrm{NaCl}$,

$5431 \% \mathrm{NP} 40$, protease inhibitor) for $10 \mathrm{~min}$ on ice. Lysed samples were centrifuged and incubated

544 for $3 \mathrm{~h}$ with Pierce Protein A/G Magnetic beads (88802) which were pre-incubated over night

545 with V5 antibody (Cell signaling E9H80; $5 \mu$ g of primary antibody per $10 \mu$ of beads per

546 sample).

547 MaMTH assay. Human IFITM proteins and SARS-CoV-2 viral proteins were cloned into

548 MaMTH N-term tagged Prey and C-term tagged Bait vectors respectively using Gateway

549 cloning technology (ThermoFisher). Correctness of recombined insertions was confirmed by

550 Sanger sequencing (Eurofins). The Mammalian Membrane Two-Hybrid (MaMTH) Assay has

551 been performed as previously described ${ }^{23,44}$. HEK293T B0166 Gaussia luciferase reporter cells

552 were co-transfected in 96-well plates with $25 \mathrm{ng}$ SARS-CoV-2 protein Bait and $25 \mathrm{ng}$ IFITM

553 or control protein Prey MaMTH vectors in triplicates using PEI transfection reagent. Ga14

554 (transcription factor) as well as EGFR Bait with SHC1 Prey served as positive controls,

555 whereas SARS-CoV-2 Bait proteins with Pex7 Prey were used as negative controls. The

556 following day, Bait protein expression was induced with $0.1 \mu \mathrm{g} / \mathrm{ml}$ doxycycline. Cell-free 
supernatants were harvested 2 days post-transfection and the released Gaussia reporter was measured $1 \mathrm{~s}$ after injecting $20 \mathrm{mM}$ coelenterazine substrate using an Orion microplate

559 luminometer. To determine the level of protein interaction, Gaussia values were normalized to 560 Pex7 Prey negative control for each Bait. To determine Bait and Prey protein expression levels, 561 HEK293T B0166 transfected and treated in the same manner were harvested two days post562 transfection and lysed in Co-IP buffer (150 mM NaCl, $50 \mathrm{mM}$ HEPES, $5 \mathrm{mM}$ EDTA, 0.10\% $563 \mathrm{NP} 40,0.5 \mathrm{mM}$ sodium orthovanadate, $0.5 \mathrm{mM} \mathrm{NaF}$, protease inhibitor cocktail from Roche) and reduced in the presence of $\beta$-mercaptoethanol by boiling at $95^{\circ} \mathrm{C}$ for $10 \mathrm{~min}$. Proteins were 565 separated in 4 to $12 \%$ Bis-Tris gradient acrylamide gels (Invitrogen), blotted onto 566 polyvinylidene difluoride (PVDF) membrane, blocked in 5\% milk and probed with rabbit anti567 V5 (Cell Signaling \#13202), mouse anti-FLAG (Sigma \#F1804) and rat anti-GAPDH 568 (Biolegend \#607902) antibodies, followed by goat anti-mouse, anti-rabbit and anti-rat 569 secondary fluorescent antibodies (LI-COR). Membranes were scanned with LI-COR Odyssey 570 reader.

571 Statistics. Statistical analyses were performed using GraphPad PRISM 8 (GraphPad Software).

572 P-values were determined using a two-tailed Student's t test with Welch's correction. Unless

573 otherwise stated, data are shown as the mean of at least three independent experiments \pm SEM.

574 Significant differences are indicated as: ${ }^{*}, \mathrm{p}<0.05 ; * *, \mathrm{p}<0.01 ; * * *, \mathrm{p}<0.001$. Statistical 575 parameters are specified in the figure legends. 


\section{References}

577 1. Ksiazek, T. G. et al. A novel coronavirus associated with severe acute respiratory

578 syndrome. New England Journal of Medicine 348, 1953-1966 (2003).

5792 2. Bermingham, A. et al. Severe respiratory illness caused by a novel coronavirus, in a

580 patient transferred to the United Kingdom from the Middle East, September 2012.

581 Eurosurveillance 17, (2012).

582 3. Al-Rohaimi, A. H. \& Al Otaibi, F. Novel SARS-CoV-2 outbreak and COVID19

583 disease; a systemic review on the global pandemic. Genes Dis 7, 491-501 (2020).

584 4. Sa Ribero, M., Jouvenet, N., Dreux, M. \& Nisole, S. Interplay between SARS-CoV-2 585 and the type I interferon response. PLoS pathogens 16, e1008737 (2020).

586 5. Zhao, X., Li, J., Winkler, C. A., An, P. \& Guo, J. T. IFITM genes, variants, and their 587 roles in the control and pathogenesis of viral infections. Frontiers in Microbiology vol. 10 588 (2019).

589 6. Diamond, M. S. \& Farzan, M. The broad-spectrum antiviral functions of IFIT and 590 IFITM proteins. Nature Reviews Immunology vol. 13 46-57 (2013).

591 7. Bailey, C. C., Zhong, G., Huang, I.-C. \& Farzan, M. IFITM-Family Proteins: The 592 Cell's First Line of Antiviral Defense. Annual Review of Virology 1, 261-283 (2014).

5938 8. Perreira, J. M., Chin, C. R., Feeley, E. M. \& Brass, A. L. IFITMs restrict the 594 replication of multiple pathogenic viruses. Journal of Molecular Biology vol. 425 (Academic 595 Press, 2013).

5969 9. Shi, G. et al. Opposing activities of IFITM proteins in SARS-CoV-2 infection. EMBO $597 J$ e106501 (2020) doi:10.15252/embj.2020106501.

598 10. Smith, S. E., Weston, S., Kellam, P. \& Marsh, M. IFITM proteins - Cellular 599 inhibitors of viral entry. Current Opinion in Virology vol. 4 (Elsevier B.V., 2014).

600 11. Huang, I. C. et al. Distinct patterns of IFITM-mediated restriction of filoviruses, 601 SARS coronavirus, and influenza A virus. PLoS Pathogens 7, (2011).

602 12. Zani, A. \& Yount, J. S. Antiviral Protection by IFITM3 In Vivo. Current Clinical 603 Microbiology Reports vol. 5 229-237 (2018).

604 13. Li, K. et al. IFITM Proteins Restrict Viral Membrane Hemifusion. PLoS Pathogens 9, 605 (2013).

606 14. Shi, G., Schwartz, O. \& Compton, A. A. More than meets the I: The diverse antiviral 607 and cellular functions of interferon-induced transmembrane proteins. Retrovirology vol. 14 608 (2017).

609 15. Wrensch, F., Winkler, M. \& Pöhlmann, S. IFITM proteins inhibit entry driven by the 610 MERS-Coronavirus Spike protein: Evidence for Cholesterol-Independent Mechanisms.

611 Viruses 6, 3683-3698 (2014).

612 16. Nchioua, R. et al. SARS-CoV-2 Is Restricted by Zinc Finger Antiviral Protein despite 613 Preadaptation to the Low-CpG Environment in Humans. mBio 11, 16 (2020). 
614 17. Shang, J. et al. Cell entry mechanisms of SARS-CoV-2. PNAS 117, 11727-11734

615 (2020).

616 18. Zhao, X. et al. Interferon induction of IFITM proteins promotes infection by human

617 coronavirus OC43. Proceedings of the National Academy of Sciences of the United States of

618 America 111, 6756-6761 (2014).

619 19. Cavrois, M., De Noronha, C. \& Greene, W. C. A sensitive and specific enzyme-based 620 assay detecting HIV-1 virion fusion in primary T lymphocytes. Nature biotechnology 20,

$621 \quad 1151-4$ (2002).

622 20. Buchrieser, J. et al. Syncytia formation by SARS-CoV-2-infected cells. EMBO J 39, 623 e106267 (2020).

624 21. Yánez, D. C., Ross, S. \& Crompton, T. The IFITM protein family in adaptive 625 immunity. Immunology vol. 159 (Blackwell Publishing Ltd, 2020).

626 22. Hampp, S. et al. DNA damage tolerance pathway involving DNA polymerase ? and 627 the tumor suppressor p53 regulates DNA replication fork progression. Proceedings of the 628 National Academy of Sciences 113, E4311-E4319 (2016).

629 23. Petschnigg, J. et al. The mammalian-membrane two-hybrid assay (MaMTH) for 630 probing membrane-protein interactions in human cells. Nature Methods 11, 585-592 (2014).

631 24. Schütz, D. et al. Peptide and peptide-based inhibitors of SARS-CoV-2 entry. Adv 632 Drug Deliv Rev 167, 47-65 (2020).

633 25. Xia, S. et al. Inhibition of SARS-CoV-2 (previously 2019-nCoV) infection by a 634 highly potent pan-coronavirus fusion inhibitor targeting its spike protein that harbors a high 635 capacity to mediate membrane fusion. Cell Res 30, 343-355 (2020).

636 26. Krüger, J. et al. Remdesivir but not famotidine inhibits SARS-CoV-2 replication in 637 human pluripotent stem cell-derived intestinal organoids. bioRxiv 2020.06.10.144816 (2020) 638 doi:10.1101/2020.06.10.144816.

639 27. Lamers, M. M. et al. SARS-CoV-2 productively infects human gut enterocytes.

640 Science (New York, N.Y.) 369, 50-54 (2020).

641 28. Hoffmann, M. et al. SARS-CoV-2 Cell Entry Depends on ACE2 and TMPRSS2 and 642 Is Blocked by a Clinically Proven Protease Inhibitor. Cell (2020)

643 doi:10.1016/j.cell.2020.02.052.

644 29. Ramani, A. et al. $<\mathrm{scp}>\mathrm{SARS}</ \mathrm{scp}>-\mathrm{CoV}-2$ targets neurons of $3 \mathrm{D}$ human brain 645 organoids. The EMBO Journal 39, (2020).

646 30. Magadum, A. \& Kishore, R. Cardiovascular Manifestations of COVID-19 Infection. 647 Cells 9, (2020).

648 31. Bojkova, D. et al. SARS-CoV-2 infects and induces cytotoxic effects in human 649 cardiomyocytes. Cardiovasc Res 116, 2207-2215 (2020).

650 32. Shi, G. et al. Opposing activities of IFITM proteins in SARS-CoV-2 infection. 651 bioRxiv 2020.08.11.246678 (2020) doi:10.1101/2020.08.11.246678. 
652 33. Zhao, X. et al. Identification of Residues Controlling Restriction versus Enhancing

653 Activities of IFITM Proteins on Entry of Human Coronaviruses. Journal of Virology 92,

654 (2017).

655 34. Zhao, X. et al. Identification of Residues Controlling Restriction versus Enhancing

656 Activities of IFITM Proteins on Entry of Human Coronaviruses. Journal of Virology 92,

657 (2017).

658 35. Blanco-Melo, D. et al. Imbalanced host response to SARS-CoV-2 drives development 659 of COVID-19. Cell (2020) doi:10.1016/j.cell.2020.04.026.

660 36. Hadjadj, J. et al. Impaired type I interferon activity and inflammatory responses in 661 severe COVID-19 patients. Science (New York, N.Y.) (2020) doi:10.1126/science.abc6027.

662 37. Linta, L., Boeckers, T. M., Kleger, A. \& Liebau, S. Calcium activated potassium

663 channel expression during human iPS cell-derived neurogenesis. Ann Anat 195, 303-311

664 (2013).

665 38. Koepke, L. et al. An improved method for high-throughput quantification of

666 autophagy in mammalian cells. Scientific Reports 10, 1-20 (2020).

667 39. Cavrois, M., De Noronha, C. \& Greene, W. C. A sensitive and specific enzyme-based 668 assay detecting HIV-1 virion fusion in primary T lymphocytes. Nature Biotechnology (2002)

669 doi:10.1038/nbt745.

670 40. Krüger, J. et al. Drug Inhibition of SARS-CoV-2 Replication in Human Pluripotent

671 Stem Cell-Derived Intestinal Organoids. Cell Mol Gastroenterol Hepatol (2020)

672 doi:10.1016/j.jcmgh.2020.11.003.

673 41. Chen, C. \& Okayama, H. High-efficiency transformation of mammalian cells by

674 plasmid DNA. Molecular and Cellular Biology 7, 2745-2752 (1987).

675 42. Catanese, A. et al. Retinoic acid worsens ATG10-dependent autophagy impairment in

676 TBK1-mutant hiPSC-derived motoneurons through SQSTM1/p62 accumulation. Autophagy

677 15, 1719-1737 (2019).

678 43. Volcic, M. et al. Vpu modulates DNA repair to suppress innate sensing and hyper-

679 integration of HIV-1. Nature Microbiology (2020) doi:10.1038/s41564-020-0753-6.

680 44. Kmiec, D. et al. SIVcol Nef counteracts SERINC5 by promoting its proteasomal

681 degradation but does not efficiently enhance HIV-1 replication in human CD4+ T cells and

682 lymphoid tissue. PLOS Pathogens 14, e1007269 (2018).

683 45. He, S. et al. PSGL-1 blocks SARS-CoV-2 S protein-mediated virus attachment and

684 infection of target cells. bioRxiv : the preprint server for biology (Cold Spring Harbor

685 Laboratory, 2020). doi:10.1101/2020.05.01.073387. 
688 Acknowledgments. We thank K. Regensburger, S. Engelhart, M. Meyer, R. Burger, N. 689 Schrott, N. Preising and D. Krnavek for technical assistance and. The ACE2 vector and the 690 SARS-CoV-2 S-HA plasmid were provided by Shinji Makino and Stefan Pöhlmann. We thank 691 K-K. Conzelmann for providing VSV $\Delta \mathrm{G}$ and the Core Functional Peptidomics of Ulm 692 University for peptide synthesis. This study was supported by DFG grants to F.K., J.Mün., 693 D.K., K.M.J.S., D.Sa. (CRC 1279, SPP 1923, KM 5/1-1, SP1600/4-1), C.G. (GO2153/3-1) 694 EU's Horizon 2020 research and innovation program to J.M. (Fight-nCoV, 101003555), as well as the BMBF to F.K., D.Sa. and K.M.J.S. (Restrict SARS-CoV-2, protACT and IMMUNOMOD). C.P.B., C.C., and R.G. are part of and R.G. is funded by a scholarship from the International Graduate School in Molecular Medicine Ulm (IGradU).

Author Contributions. C.P.B. and R.N. performed most experiments. M.V. performed interaction assays. J.K., S.H. and A.K. provided gut organoids. C.M.S. generated most expression constructs. D.K. performed MaMTH assays. J. Mül., C.C. and J. Mün provided SARS-CoV-2. F.Z. assisted in experiments with infectious SARS-CoV-2. L.W., T.W. and R.G. provided reagents and protocols. D.Sc. performed FACS for the Vpr-BlaM assay; E.B. and J.W. performed the HEK293T GFP split fusion assay. L.K. helped with the microscopy analysis of organoids. F.D. and S.J. provided cardiomyocytes. A.C. M.S. and T.B. provided neurons. D.S., C.G., S.S. and J. Mün. provided comments and resources. K.M.J.S and F.K. conceived the study, planned experiments and wrote the manuscript. All authors reviewed and approved the manuscript.

Competing interests. The authors declare no competing interests.

Data Availability. The datasets generated during and/or analyzed during the current study are available from the corresponding authors on request. 


\section{$711 \quad$ Figure legends}

712 Fig. 1 | Opposing effects of IFITM proteins on SARS-CoV-2 infection. a, Quantification of

$713 \mathrm{VSV}(\mathrm{luc}) \Delta \mathrm{G} * \mathrm{SARS}-\mathrm{CoV}-2-\mathrm{S}$ entry by measuring luciferase activity in HEK293T cells

714 transiently expressing the indicated IFITM proteins. Bars in all panels show results of three

715 independent experiments (mean value, \pm SEM). b, Calu-3 cells treated with non-targeting

716 (CTRL) or IFITM1, 2 or 3 siRNAs or a combination of the three and infected with

$717 \mathrm{VSV}(\mathrm{luc}) \Delta \mathrm{G}^{*} \mathrm{SARS}-\mathrm{CoV}-2-\mathrm{S}$ particles. c, Quantification of RNA containing N gene

718 sequences by qRT-PCR in the supernatant of HEK293T cells transiently expressing ACE2

719 alone or together with the indicated IFITM proteins $48 \mathrm{~h}$ post-infection with SARS-CoV-2

720 (MOI 0.05). d, RNA containing $\mathrm{N}$ gene sequences levels in the supernatant of Calu-3 cells,

721 collected $48 \mathrm{~h}$ post-infection with SARS-CoV-2 (MOI 0.05). Cells were transfected with

722 control (CTRL) or IFITM1, 2 and/or 3 targeting siRNA or a combination of the three and either

723 treated with IFN- $\beta$ or left untreated as indicated. e, Cytopathic effects in Vero cells infected

724 with serial dilutions of Calu-3 supernatants from Figure 1d. Cells were stained with crystal

725 violet.

726 Fig. 2 | Role of IFITMs in SARS-CoV-2 replication in SAEC. a, Expression of IFITM1,

727 IFITM2 and IFITM3 in SAEC after stimulation with IFN- $\alpha 2(500 \mathrm{U} / \mathrm{ml}, 72 \mathrm{~h})$, IFN- $\beta$ (500

$728 \mathrm{U} / \mathrm{ml}, 72 \mathrm{~h})$ or IFN- $\gamma(200 \mathrm{U} / \mathrm{ml}, 72 \mathrm{~h})$. Immunoblots of whole cell lysates were stained with

729 anti-IFITM1, anti-IFITM2, anti-IFITM3 and anti-GAPDH. b, Expression of IFITM proteins in

730 SAEC treated with non-targeting or IFITM specific siRNAs. Cells were either stimulated with

731 IFN- $\beta(500 \mathrm{U} / \mathrm{ml}, 72 \mathrm{~h})$ or left untreated. Immunoblots of whole cell lysates were stained with

732 anti-IFITM1, anti-IFITM2, anti-IFITM3 and anti-GAPDH. c, SARS-CoV-2 N quantification

733 in the supernatant of SAEC 2 days post-infection with SARS-CoV-2 (MOI 2.5).

734 Fig. 3 | IFITM2 promotes SARS-CoV-2 entry and interacts with the Spike protein. a,

735 Intracellular RNA containing N gene sequences copy numbers in Calu-3 cells $6 \mathrm{~h}$ (left) and 24

$736 \mathrm{~h}$ (middle) post-infection with SARS-CoV-2 (MOI 0.05). Values were normalized to GAPDH 
and calculated relative to the control (set to 100\%). The right panel shows viral RNA copies in the cell culture supernatant at $24 \mathrm{~h}$ post infection. Cells were transiently transfected with siRNA either control (CTRL) or targeting IFITM1, 2, 3, or a combination of the three as indicated. Bars represent $n=1$, measured in duplicates, \pm SD. b, Proximity ligation assay between the SARS-CoV-2 Spike and IFITM proteins in Calu-3 cells infected with SARS-CoV-2 for $2 \mathrm{~h}$ at $4^{\circ} \mathrm{C}$. DAPI (blue), nuclei. PLA signal (yellow), proximity between S/IFITMs. Results represent two independent experiments done in technical duplicates. c, PLA in SAEC. Bars represent means of $n=1$ (45-70 cells) \pm SEM. DAPI (blue), nuclei. PLA signal (yellow), proximity between S/IFITMs. Scale bar, $20 \mu \mathrm{m}$. d, Relative interaction between SARS-CoV-2 Spike and human IFITM proteins measured by MaMTH protein-protein interaction assay in cotransfected HEK293T B0166 Gaussia luciferase reporter cells. Bars represent the mean of triplicate transfections performed in two independent experiments. e, Immunoprecipitation of IFITM proteins by the Spike protein. HEK293T cells were transfected with or without a construct to overexpress SARS-CoV-2 S (indicated with a + or a -) and IFITM1, IFITM2 or IFITM3. $24 \mathrm{~h}$ post transfection, cells were harvested and SARS-CoV-2 Spike was immunoprecipitated. WCL, whole cell lysates.

Fig. 4 | Impact of IFITMs on the ACE2-SARS-CoV-2 S proximity. a, PLA between SARSCoV-2 Spike and ACE2 in Calu-3 depleted of IFITM1, IFITM2 or IFITM3 and infected with genuine SARS-CoV-2. Lines represent means of $n=2$ (a) $n=3$ (b) (60-100 cells) \pm SEM. b, PLA between Spike and ACE2 in Calu-3 cells depleted of IFITM2 and infected with SARS-CoV-2 virus on ice for $2 \mathrm{~h}$ and then incubated for $15 \mathrm{~min}$ at $37^{\circ} \mathrm{C}$. Lines represent means of $\mathrm{n}=3(200-$ 300 cells) \pm SEM. c, PLA assay between Spike and RAB5A in Calu-3 cells infected as in c. Lines represent means of $\mathrm{n}=2$ (130-200 cells) \pm SEM. DAPI (blue), nuclei. PLA signal (yellow). Scale bar, $20 \mu \mathrm{m}$. d, Quantification of ACE2-Spike and Spike-RAB5 alpha proximity upon SARS-CoV-2 infection. 
762 Fig. 5 | IFITM blocking antibodies and IFITM derived peptides target the N-terminal

763 domain. a, Alignment of the amino acid sequence of human IFITM1, 2 and 3. Binding sites

764 of IFITM blocking antibodies are indicated and the region of origin of the IFITM derived

765 peptides highlighted. b, Viral N gene RNA levels in the supernatant of Calu-3 cells treated with $766 \alpha$-ACE2, $\alpha$-IFITM1, $\alpha$-IFITM2, $\alpha$-IFITM3 and $\alpha$-IFITM1-3 antibodies, collected 48 h post 767 infection (MOI 0.05). Bars represent one to two independent experiments each measured in 768 technical duplicates (mean value, \pm SEM). c, RNA containing $\mathrm{N}$ gene sequences in the supernatant of Calu-3 cells treated with IFITM-derived peptides, collected $48 \mathrm{~h}$ post infection 770 (MOI 0.05). Bars represent two to three independent experiments each measured in technical 771 duplicates (mean value, \pm SEM).

Fig. 6 | Blocking antibodies and IFITM-derived peptides treatment decrease SARS-CoVcell-derived gut organoids after stimulation with IFN- $\beta(500 \mathrm{U} / \mathrm{ml}, 72 \mathrm{~h}) \mathbf{b}$, Cell-associated viral $\mathrm{N}$ gene RNA copy numbers in organoids treated with $\alpha$-ACE2, mIFITM2 antibody blocking peptide and $\alpha$-IFITM1-3 and infected with SARS-CoV-2 (MOI 0.15).c, Immunohistochemistry of gut organoids treated as in $\mathbf{e}$ and infected with SARS-CoV-2 (MOI 0.5). Organoids were stained with anti SARS-CoV-2 N (red), E-Cadherin (green) and DAPI (blue). Scale bar, $100 \mu \mathrm{m}$ (left panel). SARS-CoV-2 N quantification of infected gut organoids treated as in e (right panel). d, Viral $\mathrm{N}$ gene RNA levels in the supernatant of SARS-CoV-2 infected cardiomyocytes (increasing MOIs as indicated), virus containing supernatants at indicated timepoints. e, Expression of IFITM1, IFITM2 and IFITM3 in cardiomyocytes infected with SARS-CoV-2. Immunoblot of whole cell lysates stained with anti-IFITM1, antiIFITM2, anti-IFITM3 and anti-GAPDH f, Viral N gene RNA levels in the supernatant of SARS-CoV-2 infected cardiomyocytes (0.05 MOI) treated with IFITM-derived peptides, collected at indicated timepoints post infection. Bars represent two independent experiments each measured in technical duplicates (mean value, \pm SEM). bql, below quantification level. 
bioRxiv preprint doi: https://doi.org/10.1101/2020.08.18.255935; this version posted December 28, 2020. The copyright holder for this preprint (which was not certified by peer review) is the author/funder, who has granted bioRxiv a license to display the preprint in perpetuity. It is made Figure 1 available under aCC-BY-NC-ND 4.0 internatipyreflip.BozZO, Nchioua et al.
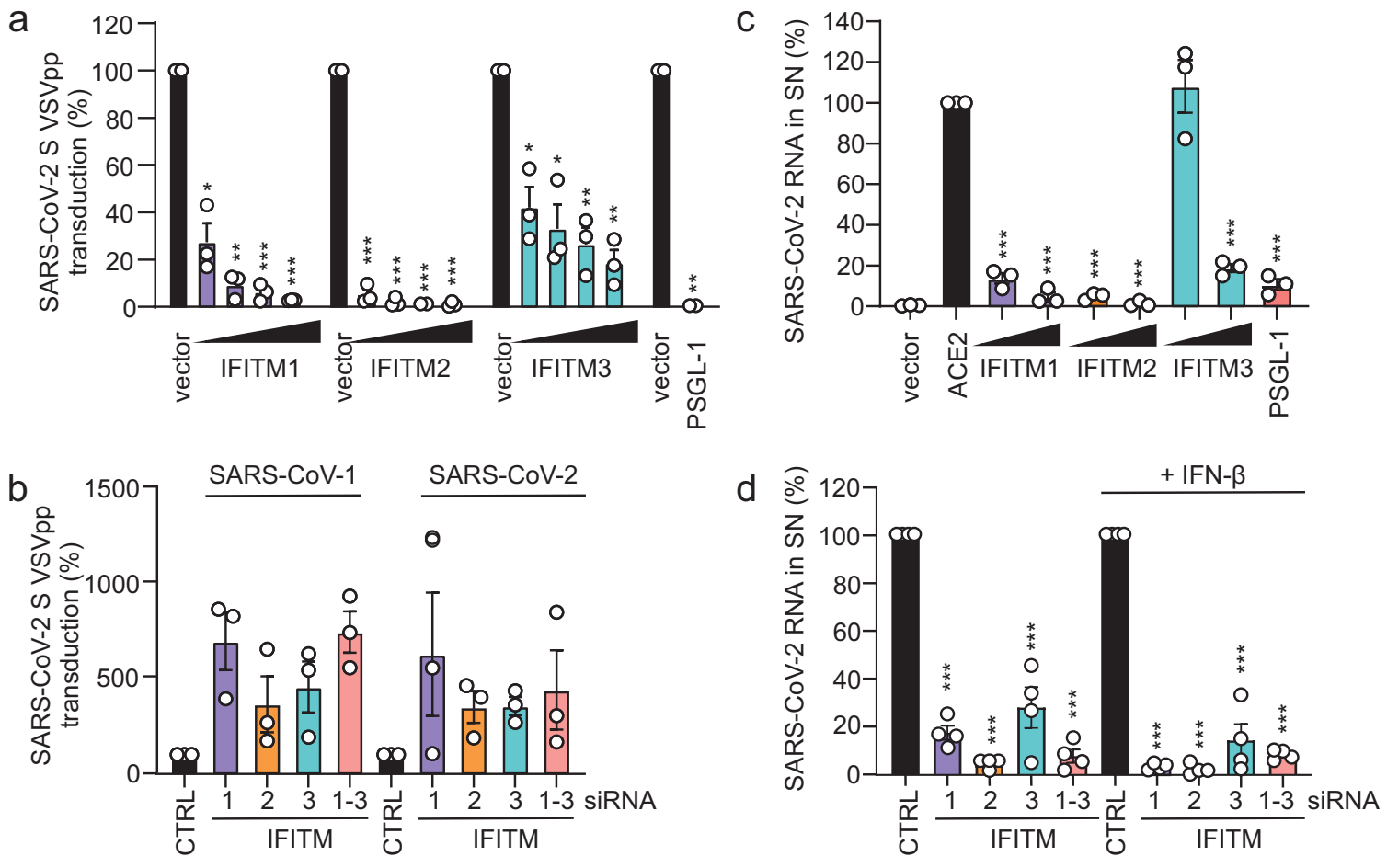

e
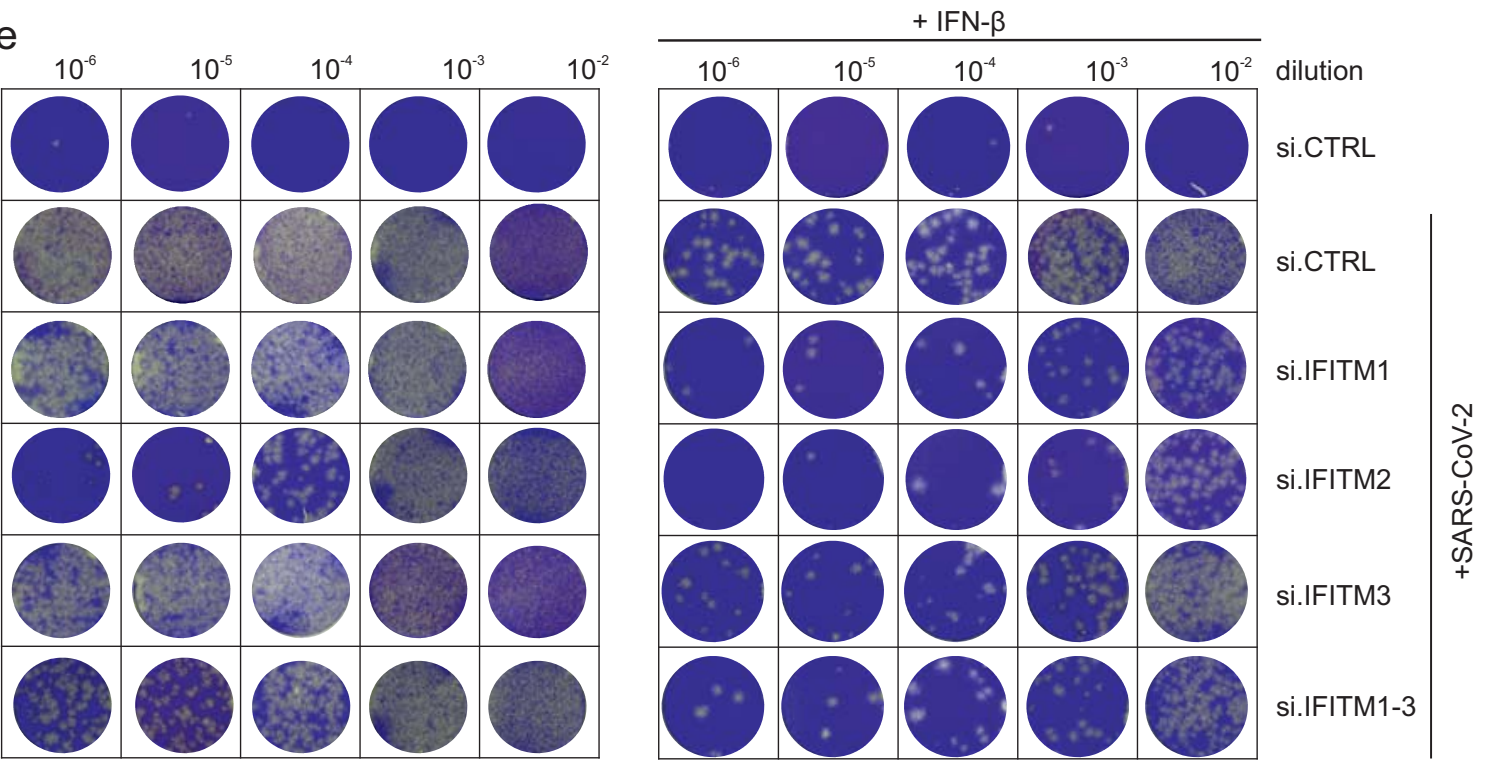
bioRxiv preprint doi: https://doi.org/10.1101/2020.08.18.255935; this version posted December 28, 2020. The copyright holder for this preprint (which was not certified by peer review) is the author/funder, who has granted bioRxiv a license to display the preprint in perpetuity. It is made Figure 2 available under aCC-BY-NC-ND 4.0 Preellifil'Bozzo, Nchioua et al.
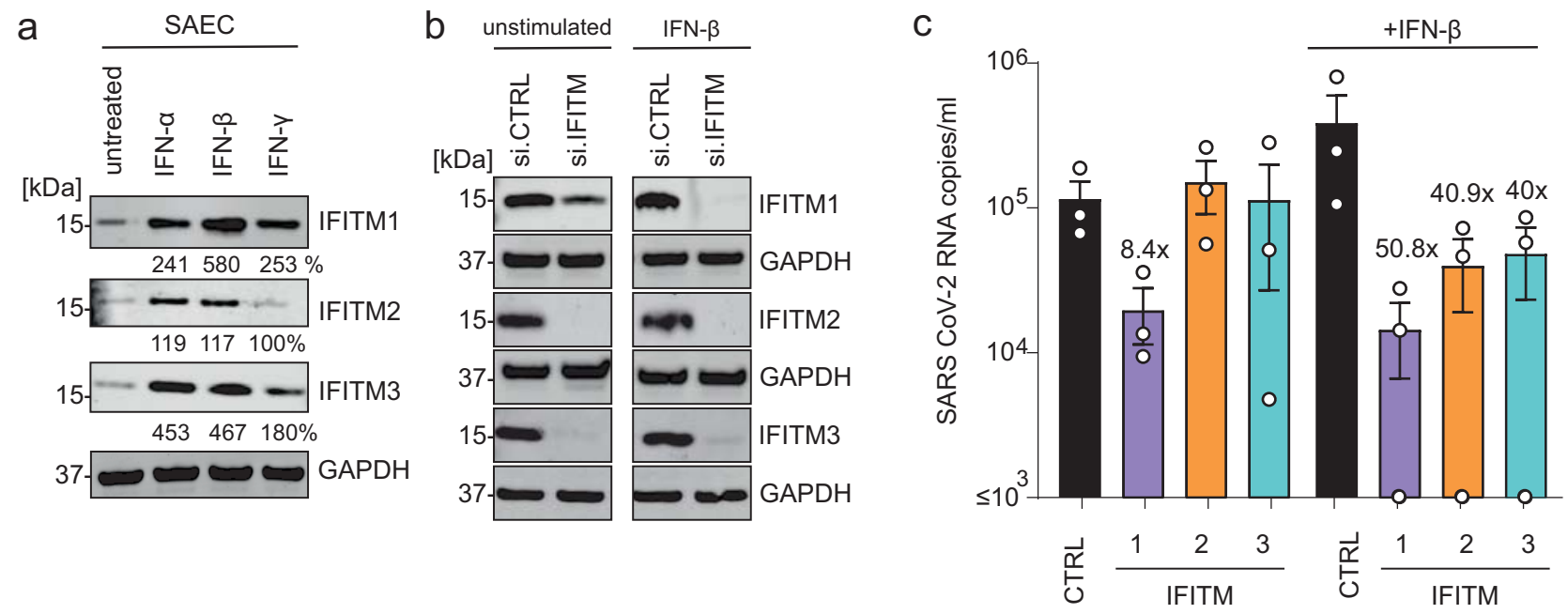


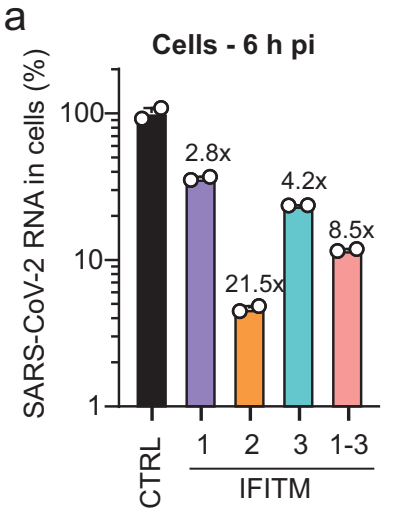

b
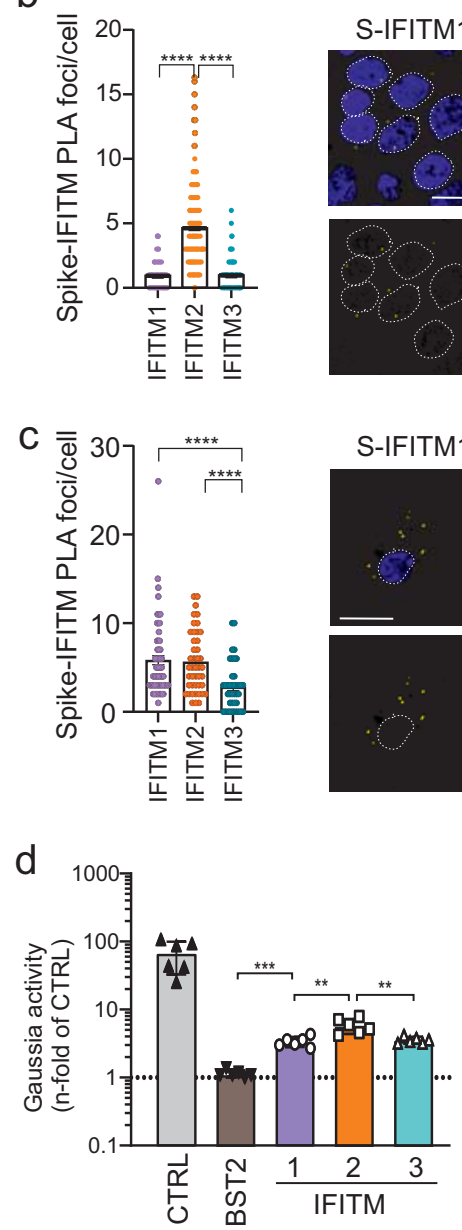


single antibody controls



$\frac{\text { e IFITM1 }}{I P .} \frac{\text { IFITM2 }}{I P .} \frac{\text { IFITM3 }}{I P .}$ WCL: anti-Spike WCL: anti-Spike WCL: anti-Spike
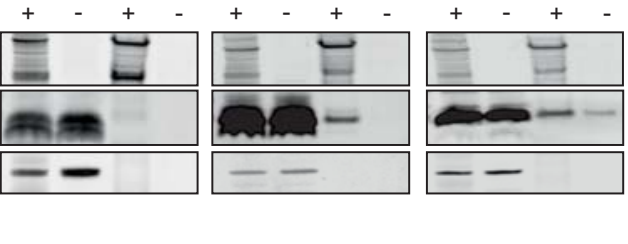

ARS-CoV-2

Spike

IFITM

GAPDH 
bioRxiv preprint doi: https://doi.org/10.1101/2020.08.18.255935; this version posted December 28,2020 . The copyright holder for this preprint (which was not certified by peer review) is the author/funder, who has granted bioRxiv a license to display the preprint in perpetuity. It is made Figure 4 available under aCC-BY-NC-ND 4.0 PrellifialBozzo, Nchioua et al.

a

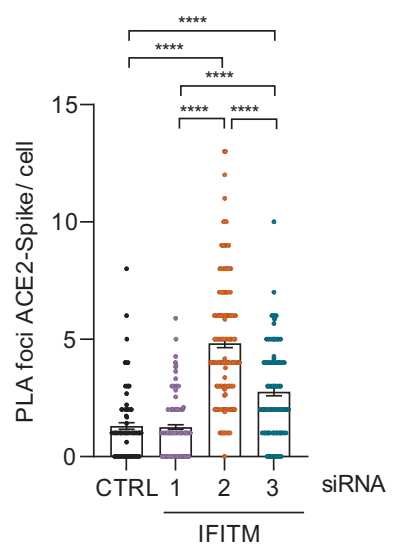

C

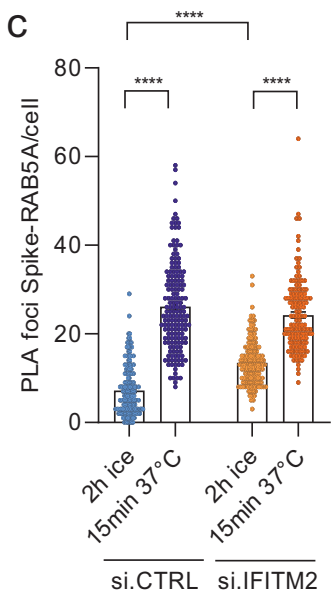

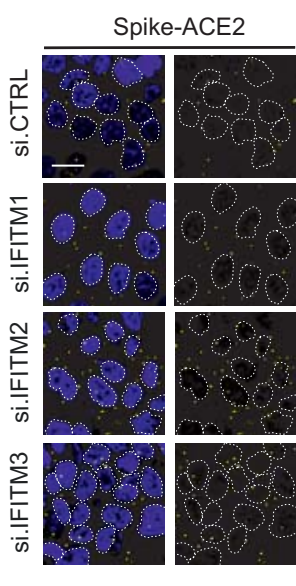
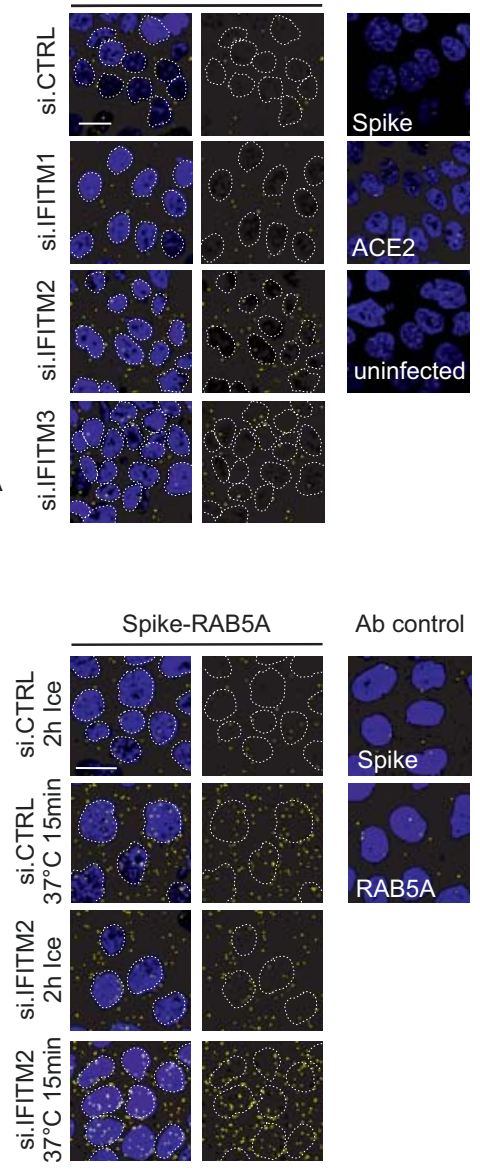

Ab control

b

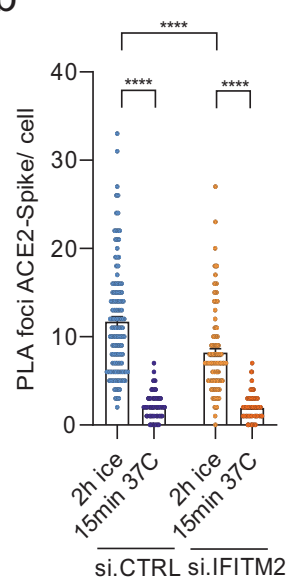

Ab control

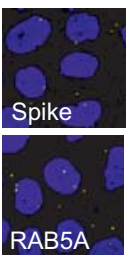

d
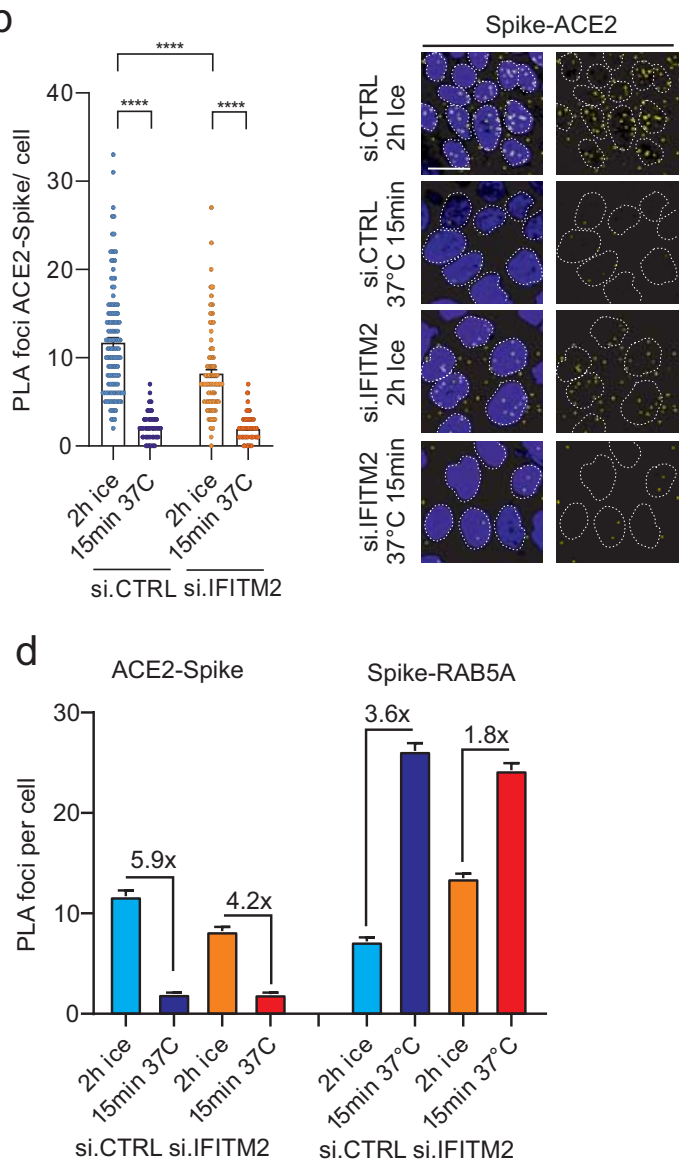

Ab control

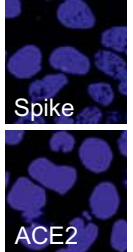


bioRxiv preprint doi: $h t t p s: / / d o i . o r g / 10.1101 / 2020.08 .18 .255935$; this version posted December 28,2020 . The copyright holder for this preprint

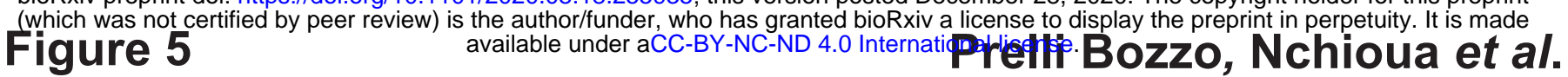

a

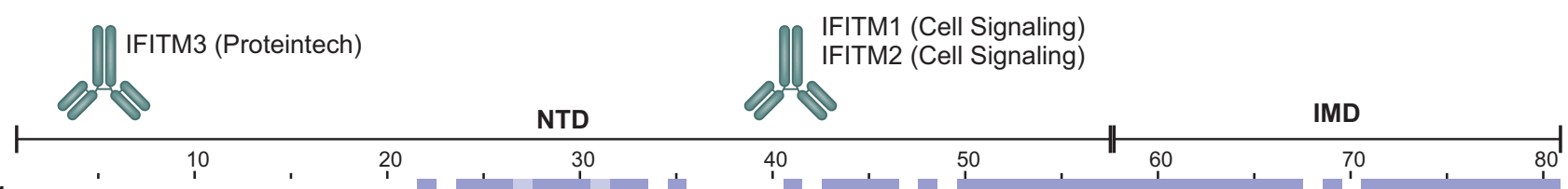

IFITM1 . . . . . . . . . . . . . . . MHKEEHEVAVLGPPPST I L'PRSTV' I N I HSETSVPDHVVWSLFNTLFLNWCCLG' I AFAY IFITM2 MNH I VQ - TF SPVNSGQPPNYEML KEEQEVAML GVPHNPAPPMSTVI HIRSETSVPDHVVWSLFNTLFMNTCCLGF I AFAY IFITM3 MNHTVQTFFSPVNSGQPPNYEMLKEEHEVAVLGAPHNPAPPTSTVIHIRSETSVPDHVVWSLFNTLFMNPCCLGFIAFAY

Human IFITM2 (long): Human IFITM2 (short):

EEQEVAMLGVPHNPAPPMSTVIH Human IFITM3:

QEVAMLGVPHNPAPPMSTVIH EEHEVAVLGAPHNPAPPTSTVIH

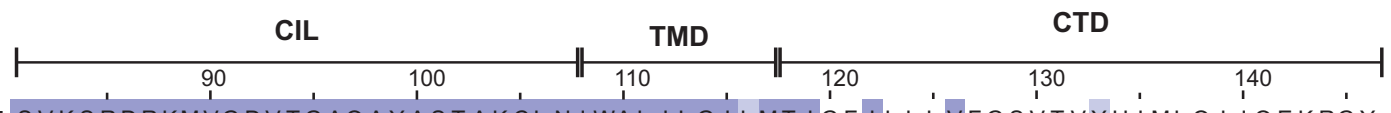

IFITM1 SVKSRDRKMVGDVTGAQAYASTAKCLN I WAL I LG ILMT I GFILLL'VFGSVTVYHIMLQIIQEKRGY IFITM2 SVKSRDRKMVGDVTGAQAYASTAKCL N I WAL I LG IFMT I LL I I I PVLVVQA - QR - . - . - . - . IFITM3 SVKSRDRKMVGDVTGAQAYASTAKCLN I WAL I LG ILMT I LL IVIPVL I FQA - YG . . . . . . . .

b

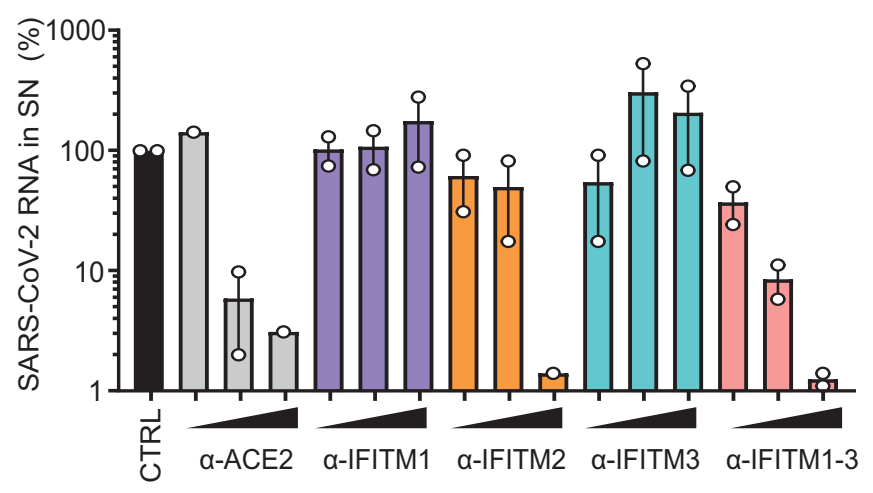

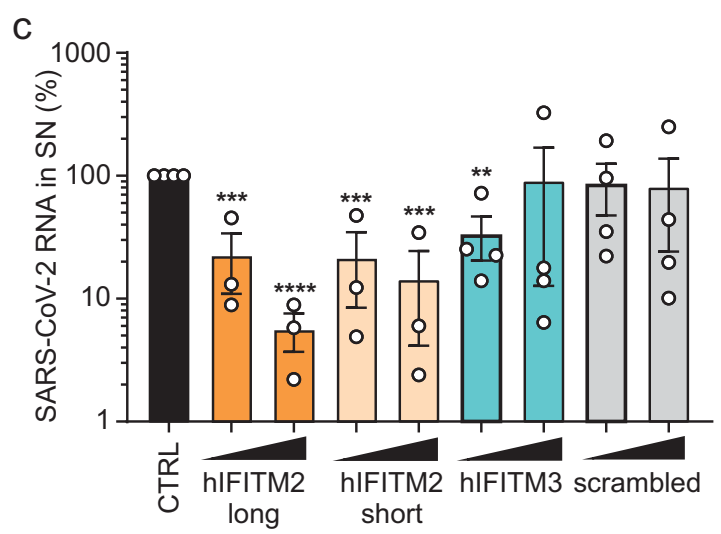


a
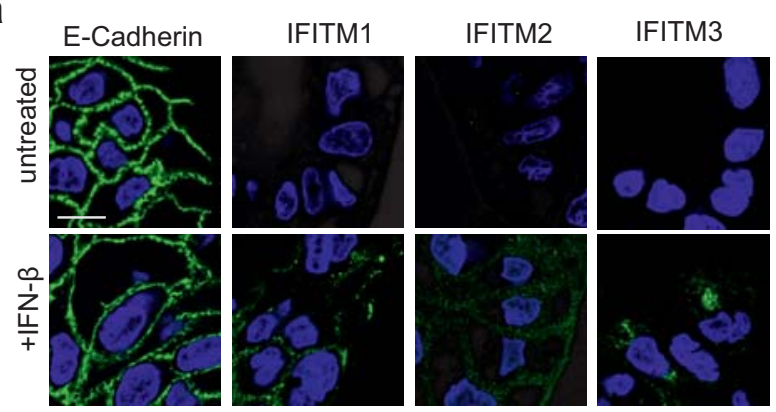

C

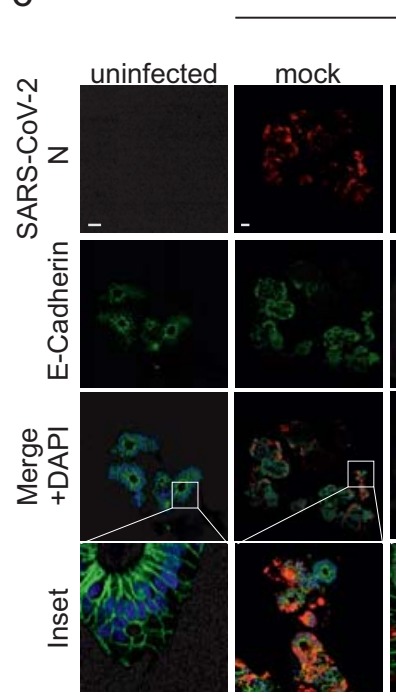

+SARS-CoV-2
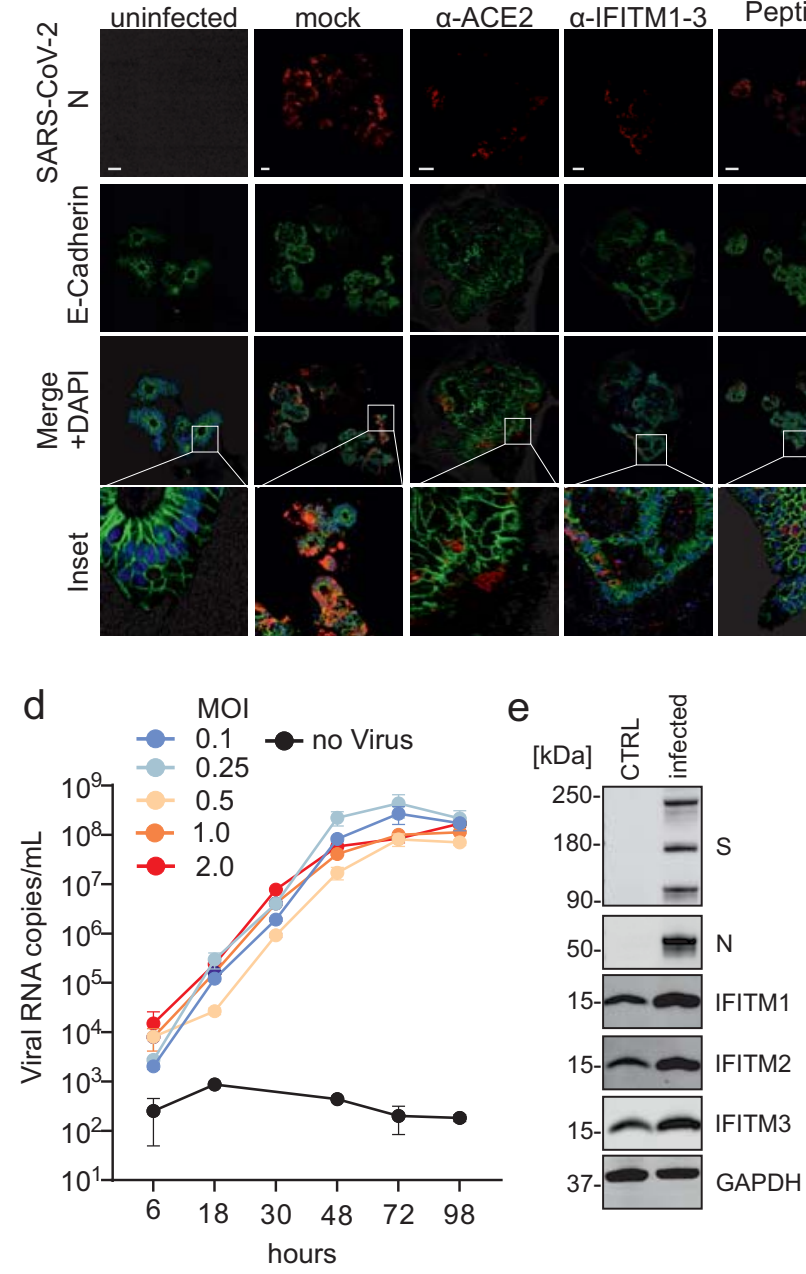

b
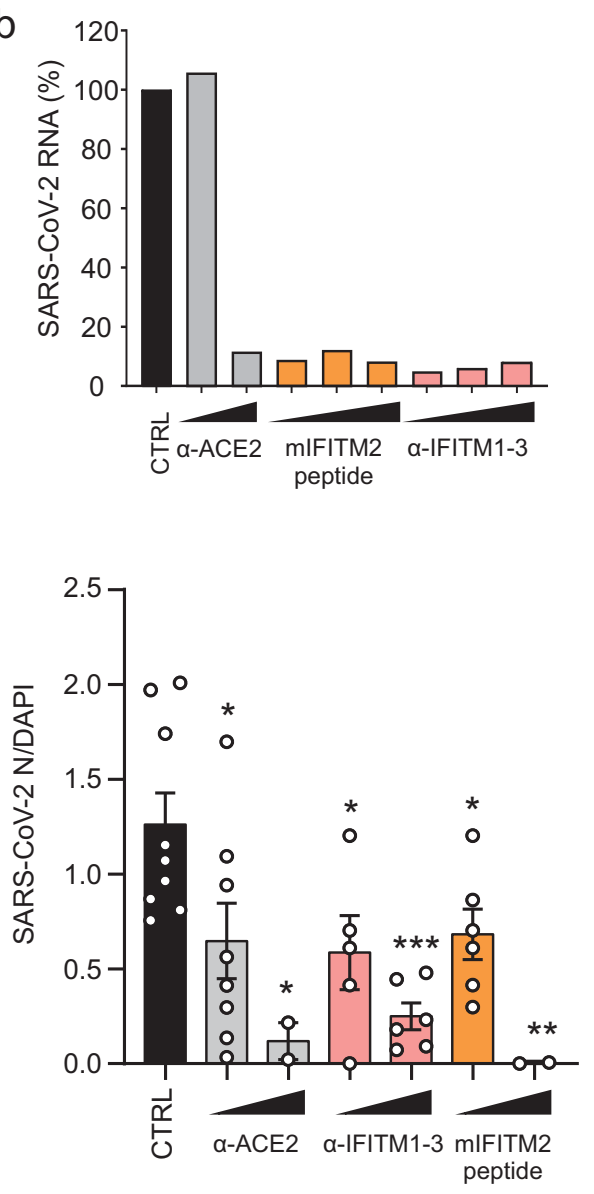

f

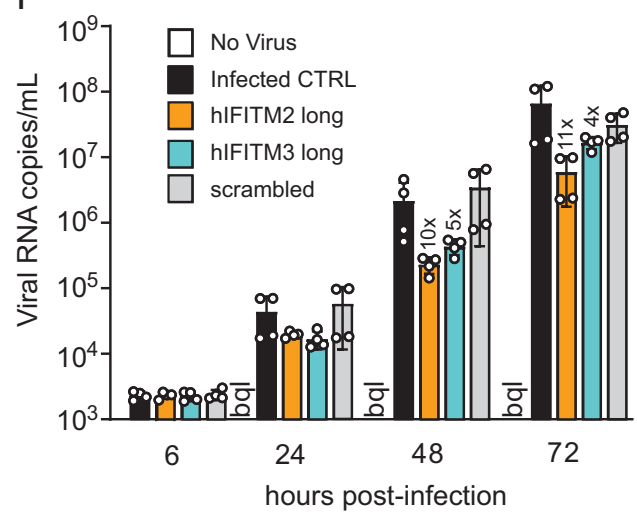

